\title{
Randomly Stopped Nonlinear Fractional Birth Processes ${ }^{\text {tr }}$
}

\author{
Enzo Orsingher ${ }^{1, *}$, Federico Polito ${ }^{1}$
}

\begin{abstract}
We present and analyse the nonlinear classical pure birth process $\mathcal{N}(t), t>0$, and the fractional pure birth process $\mathcal{N}^{\nu}(t), t>0$, subordinated to various random times, namely the first-passage time $T_{t}$ of the standard Brownian motion $B(t), t>0$, the $\alpha$-stable subordinator $\mathcal{S}^{\alpha}(t), \alpha \in(0,1)$, and others. For all of them we derive the state probability distribution $\hat{p}_{k}(t), k \geq 1$ and, in some cases, we also present the corresponding governing differential equation.

We also highlight interesting interpretations for both the subordinated classical birth process $\hat{\mathcal{N}}(t), t>0$, and its fractional counterpart $\hat{\mathcal{N}}^{\nu}(t), t>0$ in terms of classical birth processes with random rates evaluated on a stretched or squashed time scale.

Various types of compositions of the fractional pure birth process $\mathcal{N}^{\nu}(t)$ have been examined in the last part of the paper. In particular, the processes $\mathcal{N}^{\nu}\left(T_{t}\right), \mathcal{N}^{\nu}\left(\mathcal{S}^{\alpha}(t)\right), \mathcal{N}^{\nu}\left(T_{2 \nu}(t)\right)$, have been analysed, where $T_{2 \nu}(t), t>0$, is a process related to fractional diffusion equations. Also the related process $\mathcal{N}\left(\mathcal{S}^{\alpha}\left(T_{2 \nu}(t)\right)\right)$ is investigated and compared with $\mathcal{N}\left(T_{2 \nu}\left(\mathcal{S}^{\alpha}(t)\right)\right)=\mathcal{N} \nu\left(\mathcal{S}^{\alpha}(t)\right)$. As a byproduct of our analysis, some formulae relating Mittag-Leffler functions are obtained.
\end{abstract}

Keywords: Fractional nonlinear pure birth processes, Subordination, $\alpha$-stable subordinator, Fractional derivative, First-passage time, Mittag-Leffler functions, Wright functions, Lamperti law.

\section{Introduction}

We here consider the pure birth process $\mathcal{N}(t), t>0$, (linear and nonlinear) composed with different processes like the first-passage time of Brownian motion $T_{t}$ (possibly iterated n-times), the sojourn time of Brownian motion $\Gamma_{t}$ and bridge $\mathfrak{G}_{t}$, and $\alpha$-stable processes $\mathcal{S}^{\alpha}(t)$.

The subordination of processes (first introduced by Bochner [4]) has been studied by several authors, over the years, in connection, for example, to modelling the wear of instruments during the real working time, or security trading which takes into account fluctuations of the economic activity during the time elapse $t$ (see Lee and Whitmore [12]).

The second part of the paper concerns the subordination of the fractional pure birth process $\mathcal{N}^{\nu}(t), t>0$, $0<\nu \leq 1$, with the processes $\mathcal{S}^{\alpha}(t)$ and $T_{2 \alpha}(t)$, establishes that $\mathcal{N}{ }^{\nu}\left(\mathcal{S}^{\alpha}(t)\right)=\mathcal{N}\left(T_{2 \nu}\left(\mathcal{S}^{\alpha}(t)\right)\right)$, and discuss its connection with $\mathcal{N}\left(\mathcal{S}^{\alpha}\left(T_{2 \nu}(t)\right)\right)$.

Subordinated processes connected with fractional and higher order partial differential equations are treated in numerous recent papers. Most of them concern compositions of time-continuous processes (see

\footnotetext{
放2010 Mathematics Subject Classification. Primary 60G22, 60G55.

${ }^{*}$ Corresponding author.

${ }^{1}$ Dipartimento di Scienze Statistiche, "Sapienza" Università di Roma, Piazzale Aldo Moro 5, 00185 Rome, Italy. Piazzale Aldo Moro 5, 00185. Tel: +39 06 49910585, fax: +39 06 4959241. E-mail address: enzo.orsingher@uniroma1.it.

${ }^{2}$ Dipartimento di Scienze Statistiche, "Sapienza" Università di Roma, Piazzale Aldo Moro 5, 00185 Rome, Italy. Piazzale Aldo Moro 5, 00185. Tel: +39 06 49910499, fax: +39 06 4959241. E-mail address: federico.polito@uniroma1.it.
} 
for example Baeumer et al. [2]), but also point processes (Laskin [11], Mainardi and Gorenflo [13], Uchaikin et al. [17], Beghin and Orsingher [3], Meerschaert et al. [14]).

Birth processes stopped at different random times can be useful to model branching processes under laboratory conditions. For diseases started off artificially, the spread of the infected population can be stopped when the experiment leads to convincing conclusions. The cost of the investigation can play a certain role in stopping the artificially constructed experiment. The fluctuations of the temperature during the effective time $t$ can influence the growth rapidity of cells or of bacteria and thus the population size can be thought as a function of the temperature modelled as a random time process. The same reasoning underlies experiments in physical studies on chain reactions. In the case of $\mathcal{N}\left(\Gamma_{t}\right)$, where $\Gamma_{t}$ is the sojourn time of a Brownian motion on the positive half-line, the experiment can be interrupted immediately (if it proves useless), or at the end of the time interval $[0, t]$ (in the case that no evidence can be attained in a short time).

We recall that the distribution of the nonlinear fractional birth process (with one progenitor) reads

$$
\operatorname{Pr}\left\{\mathcal{N}^{\nu}(t)=k \mid \mathcal{N}^{\nu}(0)=1\right\}= \begin{cases}\prod_{j=1}^{k-1} \lambda_{j} \sum_{m=1}^{k} \frac{E_{\nu, 1}\left(-\lambda_{m} t^{\nu}\right)}{\prod_{l=1, l \neq m}^{k}\left(\lambda_{l}-\lambda_{m}\right)}, & k>1, \\ E_{\nu, 1}\left(-\lambda_{1} t^{\nu}\right), & k=1,\end{cases}
$$

where

$$
E_{\nu, \gamma}(x)=\sum_{h=0}^{\infty} \frac{x^{h}}{\Gamma(\nu h+\gamma)},
$$

is the Mittag-Leffler function and $\lambda_{k}, k \geq 1$, are the birth rates (see Orsingher and Polito [15]).

For $\lambda_{k}=\lambda \cdot k$ (fractional linear birth process), formula (1.1) takes the simple form

$$
\operatorname{Pr}\left\{N^{\nu}(t)=k \mid N^{\nu}(0)=1\right\}=\sum_{m=1}^{k}\left(\begin{array}{c}
k-1 \\
m-1
\end{array}\right)(-1)^{m-1} E_{\nu, 1}\left(-\lambda m t^{\nu}\right), \quad k \geq 1, t>0 .
$$

For $\nu=1$, we retrieve from (1.1) and (1.3) the classical distributions of nonlinear and linear pure birth process, by taking into account that $E_{1,1}(x)=e^{x}$.

The simplest subordinator considered is the first-passage time

$$
T_{t}=\inf \{s: B(s)=t\},
$$

where $B$ is a standard Brownian motion, independent of the birth process considered. For us it is relevant that the probability density of (1.4)

$$
q(t, s) d s=\operatorname{Pr}\left\{T_{t} \in d s\right\}
$$

satisfies the following equation

$$
\frac{\partial^{2}}{\partial t^{2}} q(t, s)=2 \frac{\partial}{\partial s} q(t, s), \quad t>0, s>0
$$

as a direct check shows.

In view of (1.6) we can establish the following relation between the state probabilities

$$
\hat{p}_{k}^{\nu}(t)=\operatorname{Pr}\left\{\mathcal{N}^{\nu}\left(T_{t}\right)=k\right\}
$$

and (1.1):

$$
\frac{d^{2}}{d t^{2}} \hat{p}_{k}^{\nu}(t)=-2 \int_{0}^{\infty} q(t, s) \frac{d}{d s} \operatorname{Pr}\left\{\mathcal{N}^{\nu}(s)=k\right\} d s
$$


For $\nu=1$, equation (1.8) becomes the second-order difference-differential equation

$$
\frac{d^{2}}{d t^{2}} \hat{p}_{k}^{\nu}(t)=2\left[\lambda_{k} \hat{p}_{k}^{\nu}(t)-\lambda_{k-1} \hat{p}_{k-1}^{\nu}(t)\right], \quad k \geq 1 .
$$

Furthermore, for $\nu=1$, the probability distribution (1.7) can be worked out explicitely and becomes

$$
\hat{p}_{k}(t)= \begin{cases}\prod_{j=1}^{k-1} \lambda_{j} \sum_{m=1}^{k} \frac{e^{-t \sqrt{2 \lambda_{m}}}}{\prod_{l=1, l \neq m}^{k}\left(\lambda_{l}-\lambda_{m}\right)}, & k>1, t>0, \\ e^{-t \sqrt{2 \lambda_{1}}}, & k=1, t>0 .\end{cases}
$$

For $0<\nu<1$, in light of the well-known integral representation of the Mittag-Leffler function

$$
E_{\nu, 1}\left(-\lambda t^{\nu}\right)=\frac{\sin \nu \pi}{\pi} \int_{0}^{\infty} \frac{r^{\nu-1} e^{-r \lambda^{\frac{1}{\nu}} t}}{r^{2 \nu}+2 r^{\nu} \cos \nu \pi+1} d r, \quad \nu \in(0,1),
$$

we obtain several different representations of the distributions of the subordinated processes.

For $\nu=1 / 2$, we have the following result

$$
\hat{p}_{k}^{\frac{1}{2}}(t)=\frac{\sqrt{2}}{\pi} \int_{0}^{\infty} \frac{1}{\left(\frac{w^{2}}{2}+1\right)} \operatorname{Pr}\left\{\mathcal{N}_{w}(t)=k \mid \mathcal{N}_{w}(0)=1\right\} d w,
$$

which shows that $\mathfrak{N}^{1 / 2}\left(T_{t}\right)$ is equivalent in distribution to a fractional pure birth process (denoted by $\mathcal{N}_{W}(t)$ ) with rates $\lambda_{k} \cdot W$, where $W$ is a folded Cauchy distribution with scale parameter equal to $\sqrt{2}$.

We have also that

$$
\hat{p}_{k}^{\frac{1}{2}}(t)=\int_{0}^{\infty} \operatorname{Pr}\{\mathcal{N}(s)=k\} \operatorname{Pr}\{|C(\sqrt{2} t)| \in d s\} .
$$

In other words, $\mathcal{N}^{1 / 2}\left(T_{t}\right)$ is also equivalent in distribution to $\mathcal{N}(|C(\sqrt{2} t)|), C$ being a Cauchy process.

We generalise the previous framework by considering the iterated process

$$
\tilde{\mathcal{N}}^{\nu}(t)=\mathcal{N}^{\nu}\left[T_{T_{\cdot \cdot T_{t}^{n}}^{2}}^{1}\right], \quad t>0,
$$

where $T_{t}^{1}, \ldots, T_{t}^{n}$, are independent first-passage times and

$$
T_{T_{\cdot \cdot T_{t}^{n}}^{j+1}}^{j}=\inf \left\{s: B^{j}(s)=T_{T_{\cdot \cdot T_{t}^{n}}^{j+2}}^{j+1}\right\}, \quad j=1, \ldots,(n-1),
$$

where $B^{j}(t), t>0,1 \leq j \leq n$, are independent Brownian motions. In particular, for $\nu=1$ we show that the state probabilities

$$
\operatorname{Pr}\left\{\tilde{\mathcal{N}}^{1}(t)=k\right\}=\tilde{p}_{k}^{1}(t)
$$

satisfy the $2^{n}$ th order equations

$$
\frac{d^{2^{n}}}{d t^{2^{n}}} \tilde{p}_{k}^{1}(t)=2^{2^{n}-1}\left\{\lambda_{k} \tilde{p}_{k}^{1}(t)-\lambda_{k-1} \tilde{p}_{k-1}^{1}(t)\right\}
$$

The distribution $\tilde{\mathcal{N}}^{1}(t)=\tilde{\mathcal{N}}(t)$ (for short) is directly derived and reads

$$
\tilde{p}_{k}(t)= \begin{cases}\prod_{j=1}^{k-1} \lambda_{j} \sum_{m=1}^{k} \frac{e^{-t \lambda \lambda_{m}^{\frac{1}{2^{n}}}\left(1-\frac{1}{2^{\pi}}\right)}}{\prod_{l=1, l \neq m}^{k}\left(\lambda_{l}-\lambda_{m}\right)}, & k>1, \\ e^{-t \lambda_{1}^{\frac{1}{2^{n}}} 2^{\left(1-\frac{1}{2^{n}}\right)},} & k=1 .\end{cases}
$$


For $n \rightarrow \infty$, we obtain from (1.18) that

$$
\lim _{n \rightarrow \infty} \tilde{p}_{k}(t)= \begin{cases}e^{-2 t}, & k=1, \\ 0, & k>1 .\end{cases}
$$

In the last part of the paper we examine different types of compositions of the fractional pure birth process with positively skewed stable processes $\mathcal{S}^{\alpha}(t), t>0,0<\alpha \leq 1$. For $\alpha=\nu$, we show that

$$
\mathcal{N}^{\nu}\left(\mathcal{S}^{\nu}(t)\right) \stackrel{\text { i.d. }}{=} \mathcal{N}\left(t \mathcal{W}_{\nu}\right), \quad 0<\nu<1 .
$$

For the stable random variables $\mathcal{S}_{1}^{\nu}, \mathcal{S}_{2}^{\nu}$, it is well-known that the ratio

$$
\mathcal{W}_{\alpha}=\left(\frac{\mathcal{S}_{1}^{\nu}}{\mathcal{S}_{2}^{\nu}}\right)^{\alpha}
$$

(sometimes called Lamperti law), has probability density equal to

$$
f_{\mathcal{W}_{\alpha}}(r)=\frac{\sin \nu \pi}{\alpha \pi} \frac{r^{\frac{\nu}{\alpha}-1}}{r^{2 \frac{\nu}{\alpha}}+2 r^{\frac{\nu}{\alpha}} \cos \nu \pi+1}, \quad r>0 .
$$

Furthermore, we show that $\mathcal{N}^{\nu}\left(\mathcal{S}^{\nu}(t)\right)=\mathcal{N}\left(T_{2 \nu}\left(\mathcal{S}^{\nu}(t)\right)\right) \stackrel{\text { i.d. }}{\neq} \mathcal{N}\left(\mathcal{S}^{\nu}\left(T_{2 \nu}(t)\right)\right)$. We are also able to prove that

$$
\mathcal{N}^{\nu}\left(T_{2 \alpha}(t)\right)=\mathcal{N}\left(T_{2 \nu}\left(T_{2 \alpha}(t)\right)\right) \stackrel{\text { i.d. }}{=} \mathcal{N}\left(T_{2 \nu \alpha}(t)\right)=\mathcal{N}^{\nu \alpha}(t) .
$$

As a byproduct of our analysis we obtain the following integral relation between Mittag-Leffler functions of different indices:

$$
\begin{aligned}
E_{\nu \alpha, 1}\left(-\lambda_{m} t^{\nu \alpha}\right) & =\frac{\sin \nu \pi}{\pi} \int_{0}^{\infty} \frac{r^{\nu-1}}{r^{2 \nu}+2 r^{\nu} \cos \nu \pi+1} E_{\alpha, 1}\left(-r \lambda_{m}^{\frac{1}{\nu}} t^{\alpha}\right) d r \\
& =\frac{\sin \alpha \pi}{\pi} \int_{0}^{\infty} \frac{r^{\alpha-1}}{r^{2 \alpha}+2 r^{\alpha} \cos \alpha \pi+1} E_{\nu, 1}\left(-r \lambda_{m}^{\frac{1}{\alpha}} t^{\nu}\right) d r, \quad 0<\alpha, \nu \leq 1 .
\end{aligned}
$$

\section{Subordinated nonlinear birth processes}

In this section we study in detail the nonlinear pure birth process stopped at $T_{t}$ and we derive the state probabilities $\hat{p}=\operatorname{Pr}\left\{\mathcal{N}\left(T_{t}\right)=k \mid \mathcal{N}(0)=1\right\}, k \geq 1$, and the corresponding governing differential equations.

We give some information about the process $\mathcal{N}(t), t>0$, evaluate explicitly its mean value $\mathbb{E} \mathcal{N}(t)$, and discuss also the linear birth process (sometimes referred as Yule-Furry process).

\subsection{Preliminaries} $322)$

The state probabilities $p_{k}(t)=\operatorname{Pr}\{\mathcal{N}(t)=k \mid \mathcal{N}(0)=1\}$ read (see e.g. Gikhman and Skorokhod [7], page

$$
p_{k}(t)= \begin{cases}\prod_{j=1}^{k-1} \lambda_{j} \sum_{m=1}^{k} \frac{e^{-\lambda_{m} t}}{\prod_{l=1, l \neq m}^{k}\left(\lambda_{l}-\lambda_{m}\right)}, & k>1, t>0, \\ e^{-\lambda_{1} t}, & k=1, t>0 .\end{cases}
$$

For the case of $n_{0}$ progenitors (see Chiang [5], page 51), formula (2.1) must be replaced by

$$
p_{k}(t)= \begin{cases}\prod_{j=n_{0}}^{k-1} \lambda_{j} \sum_{m=n_{0}}^{k} \frac{e^{-\lambda_{m} t}}{\prod_{l=n_{0}, l \neq m}^{k}\left(\lambda_{l}-\lambda_{m}\right)}, & k>n_{0}, t>0, \\ e^{-\lambda_{n_{0}} t}, & k=n_{0}, t>0 .\end{cases}
$$


We assume that $\sum_{k} 1 / \lambda_{k}=\infty$ in such a way that the process is non-exploding (see Feller [6], page 452). For a discussion on this point, consult Grimmett and Stirzaker [8], page 252. The probabilities (2.1) satisfy the following difference-differential equations:

$$
\frac{d}{d t} p_{k}(t)=-\lambda_{k} p_{k}(t)+\lambda_{k-1} p_{k-1}(t), \quad k \geq 1 .
$$

We have our first result in the next theorem.

Theorem 2.1. The mean value of the nonlinear birth process is

$$
\mathbb{E} \mathcal{N}(t)=1+\sum_{k=1}^{\infty}\left\{1-\sum_{m=1}^{k} \prod_{l=1, l \neq m}^{k} \frac{\lambda_{l}}{\lambda_{l}-\lambda_{m}} e^{-\lambda_{m} t}\right\} .
$$

Proof. From equation (2.3), we have that

$$
\sum_{k=1}^{\infty} k \frac{d}{d t} p_{k}(t)=-\sum_{k=1}^{\infty} k \lambda_{k} p_{k}(t)+\sum_{k=2}^{\infty} k \lambda_{k-1} p_{k-1}=\sum_{k=1}^{\infty} \lambda_{k} p_{k}(t) .
$$

By integrating both members in $(0, t)$, we obtain

$$
\begin{aligned}
\sum_{k=1}^{\infty} k p_{k}(t)-1 & =\sum_{k=1}^{\infty} \lambda_{k} \int_{0}^{t} p_{k}(s) d s \\
& =\lambda_{1} \int_{0}^{t} p_{1}(s) d s+\sum_{k=2}^{\infty} \lambda_{k}\left\{\int_{0}^{t} \prod_{j=1}^{k-1} \lambda_{j} \sum_{m=1}^{k} \frac{e^{-\lambda_{m} s}}{\prod_{l=1, l \neq m}^{k}\left(\lambda_{l}-\lambda_{m}\right)} d s\right\} \\
& =1-e^{-\lambda_{1} t}+\sum_{k=2}^{\infty}\left(\prod_{j=1}^{k-1} \lambda_{j} \sum_{m=1}^{k} \frac{\left(1-e^{-\lambda_{m} t}\right)}{\lambda_{m} \prod_{l=1, l \neq m}^{k}\left(\lambda_{l}-\lambda_{m}\right)}\right) \\
& =1-e^{-\lambda_{1} t}+\sum_{k=2}^{\infty} \sum_{m=1}^{k} \prod_{l=1, l \neq m}^{k} \frac{\lambda_{l}}{\lambda_{l}-\lambda_{m}}\left(1-e^{-\lambda_{m} t}\right) \\
& =1-e^{-\lambda_{1} t}+\sum_{k=2}^{\infty}\left\{1-\sum_{m=1}^{k} \prod_{l=1, l \neq m}^{k} \frac{\lambda_{l}}{\lambda_{l}-\lambda_{m}} e^{-\lambda_{m} t}\right\} \\
& =\sum_{k=1}^{\infty}\left\{1-\sum_{m=1}^{k} \prod_{l=1, l \neq m}^{k} \frac{\lambda_{l}}{\lambda_{l}-\lambda_{m}} e^{-\lambda_{m} t}\right\},
\end{aligned}
$$

so that formula (2.4) emerges. In the second-to-last step of (2.6), we applied formula (3.12) of Orsingher et al. 16] and, in the last step, we considered that, for $k=1$, the set of numbers $\{1 \leq l \leq 1, l \neq m=1\}$, is empty and the

$$
\prod_{l=1, l \neq m}^{k} \frac{\lambda_{l}}{\lambda_{l}-\lambda_{m}}
$$

is taken equal to 1 by convention. 
Remark 2.1. As a check we can extract, from (2.4), the mean value in the linear case $\lambda_{m}=m \cdot \lambda$. Since

$$
\begin{aligned}
& \sum_{m=1}^{k} \prod_{l=1, l \neq m}^{k} \frac{\lambda l}{\lambda l-\lambda m} e^{-\lambda m t}=\sum_{m=1}^{k} \frac{1 \ldots(m-1)(m+1) \ldots k}{(m-1)(m-2) \ldots 1 \cdot(-1)^{m-1} \cdot 1 \ldots(k-m)} e^{-\lambda m t} \\
& =-\sum_{m=1}^{k} \frac{k !}{m !(k-m) !}(-1)^{m} e^{-\lambda m t}=-\sum_{m=1}^{k}\left(\begin{array}{c}
k \\
m
\end{array}\right)(-1)^{m} e^{-\lambda m t}=-\left[\left(1-e^{-\lambda t}\right)^{k}-1\right],
\end{aligned}
$$

we have that

$$
1-\sum_{m=1}^{k} \prod_{l=1, l \neq m}^{k} \frac{\lambda l}{\lambda l-\lambda m} e^{-\lambda m t}=\left(1-e^{-\lambda t}\right)^{k} .
$$

From this we readily have that

$$
\mathbb{E} N(t)=1+\sum_{k=1}^{\infty}\left(1-e^{-\lambda t}\right)^{k}=1+\frac{1}{1-\left(1-e^{-\lambda t}\right)}-1=e^{\lambda t} .
$$

The aim of this section is to compose the process $\mathcal{N}(t)$ with the first-passage time $T_{t}=\inf (s: B(s)=t)$, where $B$ is a Brownian motion independent of $\mathcal{N}(t)$.

Remark 2.2. The probability density of $T_{t}=\inf \{s: B(s)=t\}, t>0$, where $B(t)$ is a standard Brownian motion, namely

$$
\operatorname{Pr}\left\{T_{t} \in d s\right\} / d s=q(t, s)=t \frac{e^{-\frac{t^{2}}{2 s}}}{\sqrt{2 \pi s^{3}}},
$$

is the solution to the Cauchy problem

$$
\left\{\begin{array}{l}
\frac{\partial^{2}}{\partial t^{2}} q(t, s)=2 \frac{\partial}{\partial s} q(t, s), \quad t>0, s>0, \\
q(0, s)=\delta(s)
\end{array}\right.
$$

as a simple check shows.

\subsection{Pure birth process stopped at $T_{t}$}

Theorem 2.2. Let $\mathcal{N}(t), t>0$ be a classical nonlinear pure birth process and let $q(t, s), s>0, t>0$, the law of $T_{t}$. The process $\hat{\mathcal{N}}(t)=\mathcal{N}\left(T_{t}\right), t>0$, has the following distribution

$$
\hat{p}_{k}(t)= \begin{cases}\prod_{j=1}^{k-1} \lambda_{j} \sum_{m=1}^{k} \frac{e^{-t \sqrt{2 \lambda_{m}}}}{\prod_{l=1, l \neq m}^{k}\left(\lambda_{l}-\lambda_{m}\right)}, & k>1, t>0, \\ e^{-t \sqrt{2 \lambda_{1}}}, & k=1, t>0,\end{cases}
$$

and mean value equal to

$$
\mathbb{E} \mathcal{N}\left(T_{t}\right)=1+\sum_{k=1}^{\infty}\left(1-\sum_{m=1}^{k} \prod_{l=1, l \neq m}^{k} \frac{\lambda_{l}}{\lambda_{l}-\lambda_{m}} e^{-t \sqrt{2 \lambda_{m}}}\right) .
$$

The distribution (2.13) is non-exploding under the condition that $\sum_{k} 1 / \lambda_{k}=\infty$. 
Proof. The state probabilities are derived by straight calculations and by resorting to the Laplace transform of $q(t, s)$ which reads

$$
\int_{0}^{\infty} e^{-\gamma s} q(t, s) d s=\int_{0}^{\infty} e^{-\gamma s} \frac{t e^{-\frac{t^{2}}{2 s}}}{\sqrt{2 \pi s^{3}}} d s=e^{-t \sqrt{2 \gamma}} .
$$

We treat the case $k>1$ as follows. The case $k=1$ is analogous.

$$
\begin{aligned}
\hat{p}_{k}(t) & =\operatorname{Pr}\left\{\mathcal{N}\left(T_{t}\right)=k \mid \mathcal{N}(0)=1\right\}=\int_{0}^{\infty} p_{k}(s) q(t, s) d s \\
& =\int_{0}^{\infty} \prod_{j=1}^{k-1} \lambda_{j} \sum_{m=1}^{k} \frac{e^{-\lambda_{m} s}}{\prod_{l=1, l \neq m}^{k}\left(\lambda_{l}-\lambda_{m}\right)} \frac{t e^{-\frac{t^{2}}{2 s}}}{\sqrt{2 \pi s^{3}}} d s=\prod_{j=1}^{k-1} \lambda_{j} \sum_{m=1}^{k} \frac{1}{\prod_{l=1, l \neq m}^{k}\left(\lambda_{l}-\lambda_{m}\right)} e^{-t \sqrt{2 \lambda_{m}}} .
\end{aligned}
$$

In view of Theorem 2.1, we can evaluate the mean value

$$
\mathbb{E} \mathcal{N}\left(T_{t}\right)=\int_{0}^{\infty} \mathbb{E} \mathcal{N}(s) \frac{t e^{-\frac{t^{2}}{2 s}}}{\sqrt{2 \pi s^{3}}} d s=1+\sum_{k=1}^{\infty}\left(1-\sum_{m=1}^{k} \prod_{l=1, l \neq m}^{k} \frac{\lambda_{l}}{\lambda_{l}-\lambda_{m}} e^{-t \sqrt{2 \lambda_{m}}}\right) .
$$

In the linear case (2.14) can be written as

$$
\mathbb{E} \mathcal{N}\left(T_{t}\right)=\sum_{k=0}^{\infty} \sum_{m=0}^{k}(-1)^{m} e^{-t \sqrt{2 \lambda m}}
$$

On the other side, this sum diverges because

$$
\mathbb{E} \mathcal{N}\left(T_{t}\right)=\int_{0}^{\infty} e^{\lambda s} \operatorname{Pr}\left\{T_{t} \in d s\right\}=\infty .
$$

Remark 2.3. Note that $\forall t, \hat{p}_{k}(t), k \geq 1$ is a proper probability distribution because of the composition $\hat{\mathcal{N}}(t)=\mathcal{N}\left(T_{t}\right)$. The process can be appropriately interpreted by rewriting (2.16) as follows

$$
\begin{aligned}
\hat{p}_{k}(t) & =\prod_{j=1}^{k-1} \lambda_{j} \sum_{m=1}^{k} \frac{1}{\prod_{l=1, l \neq m}^{k}\left(\lambda_{l}-\lambda_{m}\right)} \int_{0}^{\infty} e^{-\lambda_{m} s} \frac{t e^{-\frac{t^{2}}{2 s}}}{\sqrt{2 \pi s^{3}}} d s \\
& =\int_{0}^{\infty} \prod_{j=1}^{k-1} \vartheta \lambda_{j} \sum_{m=1}^{k} \frac{1}{\prod_{l=1, l \neq m}^{k}\left(\vartheta \lambda_{l}-\vartheta \lambda_{m}\right)} e^{-\lambda_{m} \vartheta t^{2}} \frac{e^{-\frac{1}{2 \vartheta}}}{\sqrt{2 \pi \vartheta^{3}}} d \vartheta .
\end{aligned}
$$

The process $\hat{\mathcal{N}}(t), t>0$ can be viewed as a classical nonlinear pure birth process evaluated at time $t^{2}$ with random birth rates $\Theta \lambda_{k}, k \geq 1$, where $\Theta$ is an inverse Gaussian random variable with p.d.f.

$$
f_{\Theta}(\vartheta)=\frac{e^{-\frac{1}{2 \vartheta}}}{\sqrt{2 \pi \vartheta^{3}}}, \quad \vartheta \in \mathbb{R}^{+}
$$

The composition of $\mathcal{N}(t), t>0$, with $T_{t}$ leads to a second-order time derivative in the governing equations, as shown in the next theorem. 
Theorem 2.3. Let $\hat{p}_{k}(t), t>0, k \geq 1$, be the distribution of the process $\hat{\mathcal{N}}(t)=\mathcal{N}\left(T_{t}\right), t>0$, where $T_{t}$ is the first-passage time process of the standard Brownian motion, having transition density $q(t, s), s>0$, $t>0$. The state probabilities $\hat{p}_{k}(t), t>0, k \geq 1$, satisfy the following difference-differential equations

$$
\frac{d^{2}}{d t^{2}} \hat{p}_{k}(t)=2\left[\lambda_{k} \hat{p}_{k}(t)-\lambda_{k-1} \hat{p}_{k-1}(t)\right], \quad k \geq 1,
$$

where $\lambda_{k}, k \geq 1$ are the birth rates of the nonlinear classical birth process $\mathcal{N}(t), t>0$.

Proof. Since

$$
\hat{p}_{k}(t)=\int_{0}^{\infty} p_{k}(s) q(t, s) d s,
$$

by taking the second-order derivative w.r.t. $t$, in view of Remark 2.2 , we have that

$$
\begin{aligned}
\frac{d^{2}}{d t^{2}} \hat{p}_{k}(t) & =2 \int_{0}^{\infty} p_{k}(s) \frac{\partial}{\partial s} q(t, s) d s=\left.2 q(t, s) p_{k}(s)\right|_{s=0} ^{s=\infty}-2 \int_{0}^{\infty} \frac{d}{d s} p_{k}(s) q(t, s) d s \\
& =-2 \int_{0}^{\infty} q(t, s)\left[-\lambda_{k} p_{k}(s)+\lambda_{k-1} p_{k-1}(s)\right] d s=2\left[\lambda_{k} \hat{p}_{k}(t)-\lambda_{k-1} \hat{p}_{k-1}(t)\right] .
\end{aligned}
$$

In (2.24), we considered that $p_{k}(0)=0$, for $k>1$.

Remark 2.4. In the linear case, some calculations suffice to show that

$$
\hat{p}_{k}(t)=\operatorname{Pr}\left\{N\left(T_{t}\right)=k\right\}=\sum_{m=1}^{k}\left(\begin{array}{c}
k-1 \\
m-1
\end{array}\right)(-1)^{m-1} e^{-t \sqrt{2 \lambda m}}, \quad k \geq 1, t>0
$$

and the state probabilities satisfy the equation

$$
\frac{d^{2}}{d t^{2}} \hat{p}_{k}(t)=2 \lambda \hat{p}_{k}(t)-2 \lambda(k-1) \hat{p}_{k-1}(t), \quad k \geq 1 .
$$

\subsubsection{Iterated compositions}

Theorem 2.4. Let $\mathcal{N}(t), t>0$, be a classical nonlinear birth process. Let $T_{t}^{1}, T_{t}^{2}, \ldots, T_{t}^{n}, n \in \mathbb{N}$, be first-passage times of $n$ independent standard Brownian motions. The process

$$
\tilde{\mathcal{N}}(t)=\mathcal{N}\left[T_{T_{\cdot \cdot T_{t}^{n}}^{2}}^{1}\right], \quad t>0
$$

has the following distribution

$$
\tilde{p}_{k}(t)= \begin{cases}\prod_{j=1}^{k-1} \lambda_{j} \sum_{m=1}^{k} \frac{e^{-t \lambda_{m}^{\frac{1}{2^{n}}}{ }_{2}\left(1-\frac{1}{2^{n}}\right)}}{\prod_{l=1, l \neq m}^{k}\left(\lambda_{l}-\lambda_{m}\right)}, & k>1, t>0, \\ e^{-t \lambda_{1}^{\frac{1}{2^{n}}} 2^{\left(1-\frac{1}{2^{n}}\right)},}, & k=1, t>0 .\end{cases}
$$

Proof. We start by proving the case $n=2$ since the case $n=1$ is already proved in Theorem 2.2. We omit the details for the case $k=1$ and directly treat the case $k \geq 2$. We have that

$$
\int_{0}^{\infty} \hat{p}_{k}(s) q(t, s) d s=\int_{0}^{\infty} \prod_{j=1}^{k-1} \lambda_{j} \sum_{m=1}^{k} \frac{e^{-s \sqrt{2 \lambda_{m}}}}{\prod_{l=1, l \neq m}^{k}\left(\lambda_{l}-\lambda_{m}\right)} \frac{t e^{-\frac{t^{2}}{2 s}}}{\sqrt{2 \pi s^{3}}} d s
$$




$$
\begin{aligned}
& =\prod_{j=1}^{k-1} \lambda_{j} \sum_{m=1}^{k} \frac{1}{\prod_{l=1, l \neq m}^{k}\left(\lambda_{l}-\lambda_{m}\right)} \int_{0}^{\infty} e^{-s \sqrt{2 \lambda_{m}}} \frac{t e^{-\frac{t^{2}}{2 s}}}{\sqrt{2 \pi s^{3}}} d s \\
& =\prod_{j=1}^{k-1} \lambda_{j} \sum_{m=1}^{k} \frac{1}{\prod_{l=1, l \neq m}^{k}\left(\lambda_{l}-\lambda_{m}\right)} e^{-t \sqrt{2 \sqrt{2 \lambda_{m}}}} .
\end{aligned}
$$

It is now straightforward to generalise formula (2.29) for $n$ compositions, as follows

$$
\begin{aligned}
\tilde{p}_{k}(t) & =\prod_{j=1}^{k-1} \lambda_{j} \sum_{m=1}^{k} \frac{1}{\prod_{l=1, l \neq m}^{k}\left(\lambda_{l}-\lambda_{m}\right)} e^{-t \lambda_{m}^{\frac{1}{2^{n}}} 2^{\sum_{i=1}^{n} \frac{1}{2^{i}}}} \\
& =\prod_{j=1}^{k-1} \lambda_{j} \sum_{m=1}^{k} \frac{1}{\prod_{l=1, l \neq m}^{k}\left(\lambda_{l}-\lambda_{m}\right)} e^{-t \lambda_{m}^{\frac{1}{2^{n}}} 2^{\left(1-\frac{1}{2^{n}}\right)} .}
\end{aligned}
$$

When $n \rightarrow \infty$, equation (2.28) becomes

$$
\lim _{n \rightarrow \infty} \tilde{p}_{k}(t)= \begin{cases}e^{-2 t} \prod_{j=1}^{k-1} \lambda_{j} \sum_{m=1}^{k} \frac{1}{\prod_{l=1, l \neq m}^{k}\left(\lambda_{l}-\lambda_{m}\right)}=0, & k>1, \\ e^{-2 t}, & k=1,\end{cases}
$$

because of formula (3.4), page 51 of Chiang [5]. Therefore, the process (2.27) can either assume the state $k=1$ with probability $e^{-2 t}$, or explode with probability $1-e^{-2 t}$.

Theorem 2.5. Let $\tilde{p}_{k}(t), t>0, k \geq 1$, be the distribution of the process

$$
\tilde{\mathcal{N}}(t)=\mathcal{N}\left[T_{T_{\cdot \cdot T_{t}^{n}}^{2}}^{1}\right], \quad t>0 .
$$

The state probabilities $\tilde{p}_{k}(t), t>0, k \geq 1$, satisfy the following difference-differential equations

$$
\frac{d^{2^{n}}}{d t^{2^{n}}} \tilde{p}_{k}(t)=2^{2^{n}-1}\left\{\lambda_{k} \tilde{p}_{k}(t)-\lambda_{k-1} \tilde{p}_{k-1}(t)\right\}
$$

where $\lambda_{k}, k \geq 1$, are the birth rates of the nonlinear classical birth process $\mathcal{N}(t), t>0$.

Proof. For $n=1$, equations (2.33) reduce to equations (2.22). For $n=2$ we have that

$$
\begin{aligned}
\frac{d^{4}}{d t^{4}} \tilde{p}_{k}(t) & =\int_{0}^{\infty} \int_{0}^{\infty} \hat{p}_{k}\left(w_{1}\right) q\left(w_{2}, w_{1}\right) \frac{\partial^{4}}{\partial t^{4}} q\left(t, w_{2}\right) d w_{1} d w_{2} \\
& =2^{2} \int_{0}^{\infty} \int_{0}^{\infty} \hat{p}_{k}\left(w_{1}\right) \frac{\partial^{2}}{\partial w_{2}^{2}} q\left(w_{2}, w_{1}\right) q\left(t, w_{2}\right) d w_{1} d w_{2} \\
& =2^{2} \int_{0}^{\infty} \int_{0}^{\infty} \hat{p}_{k}\left(w_{1}\right) \frac{\partial^{2}}{\partial w_{2}^{2}} q\left(w_{2}, w_{1}\right) q\left(t, w_{2}\right) d w_{1} d w_{2} \\
& =2^{3} \int_{0}^{\infty} \int_{0}^{\infty} \hat{p}_{k}\left(w_{1}\right) \frac{\partial}{\partial w_{1}} q\left(w_{2}, w_{1}\right) q\left(t, w_{2}\right) d w_{1} d w_{2} \\
& =-2^{3} \int_{0}^{\infty} \int_{0}^{\infty} \frac{d}{d w_{1}} \hat{p}_{k}\left(w_{1}\right) q\left(w_{2}, w_{1}\right) q\left(t, w_{2}\right) d w_{1} d w_{2} \\
& =2^{3}\left\{\lambda_{k} \tilde{p}_{k}(t)-\lambda_{k-1} \tilde{p}_{k-1}(t)\right\} .
\end{aligned}
$$

The above reasoning can be generalised, thus arriving at equation (2.33). 


\subsection{Other compositions}

In this part we present the distributions of the classical nonlinear birth process $\mathcal{N}(t), t>0$, stopped at various random time processes, namely the sojourn time $\Gamma_{t}$ of a standard Brownian motion, the sojourn time $\mathfrak{G}_{t}$ of a standard Brownian bridge and the stable subordinator $\mathcal{S}^{\alpha}(t)$ of order $\alpha \in(0,1]$.

We start first by considering the nonlinear birth process at time

$$
\Gamma_{t}=\int_{0}^{t} I_{[0, \infty)}(B(s)) d s=\text { meas }\{s<t: B(s)>0\} .
$$

The process $\mathcal{N}\left(\Gamma_{t}\right)$, is a slowed down birth process. In the next theorem we provide its distribution.

Theorem 2.6. We have that

$$
\operatorname{Pr}\left\{\mathcal{N}\left(\Gamma_{t}\right)=k\right\}= \begin{cases}\prod_{j=1}^{k-1} \lambda_{j} \sum_{m=1}^{k} \frac{e^{-\frac{t}{2} \lambda_{m}} I_{0}\left(\frac{t}{2} \lambda_{m}\right)}{\prod_{l=1, l \neq m}^{k}\left(\lambda_{l}-\lambda_{m}\right)}, & k>1, t>0, \\ e^{-\frac{t}{2} \lambda_{1}} I_{0}\left(\frac{t}{2} \lambda_{1}\right), & k=1, t>0,\end{cases}
$$

where

$$
I_{0}(z)=\sum_{k=0}^{\infty}\left(\frac{z}{2}\right)^{2 k} \frac{1}{(k !)^{2}}
$$

is the zero-order Bessel function with imaginary argument.

Proof. The derivation of (2.36) is based on the evaluation of the following integral:

$$
\int_{0}^{t} e^{-s \lambda_{m}} \frac{d s}{\pi \sqrt{s(t-s)}}=e^{-\frac{t}{2} \lambda_{m}} I_{0}\left(\frac{t}{2} \lambda_{m}\right) .
$$

Remark 2.5. In view of the integral representation of the Bessel function

$$
I_{0}(z)=\frac{1}{2 \pi} \int_{0}^{2 \pi} e^{z \cos \vartheta} d \vartheta,
$$

we can give the following alternative, interesting representation of (2.36).

$$
\operatorname{Pr}\left\{\mathcal{N}\left(\Gamma_{t}\right)=k\right\}=\frac{1}{2 \pi} \int_{0}^{2 \pi} \operatorname{Pr}\left\{\mathcal{N}\left(t \sin ^{2} \frac{\vartheta}{2}\right)=k\right\} d \vartheta .
$$

In other words,

$$
\mathcal{N}\left(\Gamma_{t}\right) \stackrel{i . d .}{=} \mathcal{N}\left(t \sin ^{2} \frac{\Theta}{2}\right),
$$

where $\Theta$ is a random variable uniform in $[0,2 \pi]$.

Theorem 2.7. For the nonlinear birth process stopped at

$$
\mathfrak{G}_{t}=\int_{0}^{t} I_{[0, \infty)}(\bar{B}(s)) d s
$$

$\bar{B}(s), s>0$, being a Brownian bridge, we have that

$$
\operatorname{Pr}\left\{\mathcal{N}\left(\mathfrak{G}_{t}\right)=k\right\}= \begin{cases}\frac{1}{\lambda_{k} t}\left\{1-\sum_{m=1}^{k} \prod_{l=1, l \neq m}^{k}\left(\frac{\lambda_{l}}{\lambda_{l}-\lambda_{m}}\right) e^{-\lambda_{m} t}\right\}, & k>1, \\ \frac{1-e^{-\lambda_{1} t}}{\lambda_{1} t}, & k=1 .\end{cases}
$$


Proof. The calculation

$$
\operatorname{Pr}\left\{\mathcal{N}\left(\mathfrak{G}_{t}\right)=k\right\}=\prod_{j=1}^{k-1} \lambda_{j} \sum_{m=1}^{k} \frac{1}{\prod_{l=1, l \neq m}^{k}\left(\lambda_{l}-\lambda_{m}\right)} \int_{0}^{t} e^{-\lambda_{m} s} \operatorname{Pr}\left\{\mathfrak{G}_{t} \in d s\right\},
$$

is sufficient to arrive at result (2.43), once the well-known fact that (2.42) is uniformly distributed in $[0, t]$ is considered.

Remark 2.6. For the linear birth process, the distribution (2.43) takes a very simple form as the calculations below show. Since for $\lambda_{k}=\lambda \cdot k, \lambda>0$, we have that

$$
\prod_{l=1, l \neq m}^{k} \frac{\lambda_{l}}{\lambda_{l}-\lambda_{m}}=\left(\begin{array}{c}
k \\
m
\end{array}\right)(-1)^{m-1}
$$

we can write that

$$
\begin{aligned}
\operatorname{Pr}\left\{N\left(\mathfrak{G}_{t}\right)=k\right\} & =\frac{1}{\lambda k t}\left(1-\sum_{m=1}^{k}\left(\begin{array}{c}
k \\
m
\end{array}\right)(-1)^{m-1} e^{-\lambda m t}\right) \\
& =\frac{1}{\lambda k t} \sum_{m=0}^{k}\left(\begin{array}{c}
k \\
m
\end{array}\right)(-1)^{m} e^{-\lambda m t}=\frac{\left(1-e^{-\lambda t}\right)^{k}}{\lambda k t}, \quad k \geq 1 .
\end{aligned}
$$

The distribution (2.46) is logarithmic with parameter $1-e^{-\lambda t}$. In the logarithmic distribution with parameter $0<q<1$, we have that

$$
\begin{gathered}
\mathbb{E} L=-\frac{q}{(1-q) \log (1-q)} \\
\mathbb{V} \operatorname{ar} L=-\frac{q}{(1-q)^{2} \log (1-q)}\left[1+\frac{q}{\log (1-q)}\right] .
\end{gathered}
$$

In our case $q=1-e^{-\lambda t}$ so that

$$
\begin{gathered}
\mathbb{E} N\left(\mathfrak{G}_{t}\right)=\frac{e^{\lambda t}-1}{\lambda t} \\
\operatorname{Var} N\left(\mathfrak{G}_{t}\right)=\frac{e^{\lambda t}\left(e^{\lambda t}-1\right)}{\lambda t}\left[1-\frac{1-e^{-\lambda t}}{\lambda t}\right] .
\end{gathered}
$$

For large values of $t$ we have that

$$
\begin{gathered}
\mathbb{E} N\left(\mathfrak{G}_{t}\right) \sim \frac{e^{\lambda t}}{\lambda t}=\frac{\mathbb{E} N(t)}{\lambda t}, \\
\operatorname{VarN}\left(\mathfrak{G}_{t}\right) \sim \frac{e^{\lambda t}\left(e^{\lambda t}-1\right)}{\lambda t}=\frac{\mathbb{V a r} N(t)}{\lambda t} .
\end{gathered}
$$

Theorem 2.8. For the nonlinear birth process stopped at an $\alpha$-stable time $\mathcal{S}^{\alpha}(t)$ with distribution $q_{\alpha}(t, s)$ and Laplace transform $\int_{0}^{\infty} e^{-\mu s} q_{\alpha}(t, s) d s=e^{-t \mu^{\alpha}}$, we have that

$$
\operatorname{Pr}\left\{\mathcal{N}\left(\mathcal{S}^{\alpha}(t)\right)=k\right\}= \begin{cases}\prod_{j=1}^{k-1} \lambda_{j} \sum_{m=1}^{k} \frac{e^{-t \lambda_{m}^{\alpha}}}{\prod_{l=1, l \neq m}^{k}\left(\lambda_{l}-\lambda_{m}\right)}, & k>1, \\ e^{-t \lambda_{1}^{\alpha}}, & k=1 .\end{cases}
$$


Proof. The following calculation is sufficient to prove result (2.53):

$$
\operatorname{Pr}\left\{\mathcal{N}\left(S^{\alpha}(t)\right)=k\right\}=\prod_{j=1}^{k-1} \lambda_{j} \sum_{m=1}^{k} \frac{1}{\prod_{l=1, l \neq m}^{k}\left(\lambda_{l}-\lambda_{m}\right)} \int_{0}^{\infty} e^{-\lambda_{m} s} q_{\alpha}(t, s) d s .
$$

Remark 2.7. Formula (2.53) can be further worked out as follows.

$$
\begin{aligned}
\operatorname{Pr}\left\{\mathcal{N}\left(\mathcal{S}^{\alpha}(t)\right)=k\right\} & =\prod_{j=1}^{k-1} \lambda_{j} \sum_{m=1}^{k} \frac{e^{-t \lambda_{m}^{\alpha}}}{\prod_{l=1, l \neq m}^{k}\left(\lambda_{l}-\lambda_{m}\right)} \\
& \text { by exploiting the self-similarity of } \mathcal{S}^{\alpha}(t) \\
& =\prod_{j=1}^{k-1} \lambda_{j} \sum_{m=1}^{k} \frac{1}{\prod_{l=1, l \neq m}^{k}\left(\lambda_{l}-\lambda_{m}\right)} \int_{0}^{\infty} e^{-\lambda_{m} s} t^{-\frac{1}{\alpha}} q_{\alpha}\left(1, t^{-\frac{1}{\alpha}} s\right) d s \\
& =\prod_{j=1}^{k-1} \lambda_{j} \sum_{m=1}^{k} \frac{1}{\prod_{l=1, l \neq m}^{k}\left(\lambda_{l}-\lambda_{m}\right)} \int_{0}^{\infty} e^{-\lambda_{m} t^{\frac{1}{\alpha}} \zeta} q_{\alpha}(1, \zeta) d \zeta .
\end{aligned}
$$

The last result implies the following representation:

$$
\mathcal{N}\left(\mathcal{S}^{\alpha}(t)\right) \stackrel{i . d .}{=} \mathcal{N}\left(t^{\frac{1}{\alpha}} Z\right)
$$

where $Z$ has distribution $q_{\alpha}(1, \zeta), \zeta>0$.

Remark 2.8. If we assume $\alpha=1 / 2^{n}$ in the first line of (2.55), and $s=t 2^{1-\frac{1}{2^{n}}}$ in (2.28), the distribution (2.55) suggests the following unexpected relation:

$$
\operatorname{Pr}\left\{\mathcal{N}\left(\mathcal{S}^{\frac{1}{2^{n}}}(t)\right)=k\right\}=\operatorname{Pr}\left\{\mathcal{N}\left[T_{T_{\cdots \cdot T_{s}^{n}}^{2}}^{1}\right]=k\right\}, \quad k \geq 1 .
$$

Remark 2.9. Many other compositions can be envisaged and in some cases they provide curious results. For example, we consider the standard Cauchy process $C(t)$, with law $h(t, s), t>0, s \in \mathbb{R}$, satisfying the Laplace equation

$$
\frac{\partial^{2} h}{\partial t^{2}}+\frac{\partial^{2} h}{\partial s^{2}}=0
$$

We can show that $\mathcal{N}(|C(t)|), t>0$, is a birth process whose state probabilities $p_{k}^{*}(t), t>0$, satisfy the difference-differential equations

$$
\frac{d^{2}}{d t^{2}} p_{k}^{*}(t)=-\lambda_{k}^{2} p_{k}^{*}(t)+\lambda_{k-1}\left(\lambda_{k}+\lambda_{k-1}\right) p_{k-1}^{*}(t)-\lambda_{k-1} \lambda_{k-2} p_{k-2}^{*}(t)
$$

\section{Subordinated fractional birth processes}

In a previous work of us (see Orsingher and Polito [15]) we constructed and analysed a fractional (possibly nonlinear) pure birth process $\mathcal{N}^{\nu}(t), t>0, \nu \in(0,1]$ by exchanging the integer-order time derivative with the Dzhrbashyan-Caputo fractional derivative in the difference-differential equation (2.3) governing the state probabilities. We recall that the Dzhrbashyan-Caputo derivative has the form, for $0<\nu \leq 1$

$$
\frac{d^{\nu}}{d t^{\nu}} f(t)= \begin{cases}\frac{1}{\Gamma(1-\nu)} \int_{0}^{t} \frac{f^{\prime}(s)}{(t-s)^{\nu}} d s, & 0<\nu<1 \\ f^{\prime}(t), & \nu=1\end{cases}
$$


In this section we examine properties of the subordinated processes $\mathcal{N}^{\nu}\left(T_{t}\right), t>0, \mathcal{N}^{\nu}\left(T_{2 \beta}(t)\right)$, and $\mathcal{N}^{\nu}\left(\mathcal{S}^{\alpha}(t)\right)$, $t>0, \nu, \alpha, \beta \in(0,1]$, bringing to the fore some interesting relations and discussing the interpretation for the results obtained.

\subsection{Preliminaries}

The state probabilities $p_{k}^{\nu}(t)=\operatorname{Pr}\left\{\mathcal{N}^{\nu}(t)=k\right\}, k \geq 1$ of the fractional pure birth process have the following form

$$
p_{k}^{\nu}(t)= \begin{cases}\prod_{j=1}^{k-1} \lambda_{j} \sum_{m=1}^{k} \frac{E_{\nu, 1}\left(-\lambda_{m} t^{\nu}\right)}{\prod_{l=1, l \neq m}^{k}\left(\lambda_{l}-\lambda_{m}\right)}, & k>1, t>0, \\ E_{\nu, 1}\left(-\lambda_{1} t^{\nu}\right), & k=1, t>0,\end{cases}
$$

where $E_{\nu, 1}\left(-\zeta t^{\nu}\right)$ is the Mittag-Leffler function defined as

$$
E_{\nu, 1}\left(-\zeta t^{\nu}\right)=\sum_{h=0}^{\infty} \frac{\left(-\zeta t^{\nu}\right)^{h}}{\Gamma(\nu h+1)}, \quad \zeta \in \mathbb{R}, \nu>0,
$$

and with Laplace transform

$$
\int_{0}^{\infty} e^{-z t} E_{\nu, 1}\left(-\zeta t^{\nu}\right) d t=\frac{z^{\nu-1}}{z^{\nu}+\zeta}, \quad \nu>0
$$

A useful integral representation for $E_{\nu, 1}\left(-\zeta t^{\nu}\right)$ reads

$$
E_{\nu, 1}\left(-\zeta t^{\nu}\right)=\frac{\sin \nu \pi}{\pi} \int_{0}^{\infty} \frac{r^{\nu-1} e^{-r \zeta^{\frac{1}{\nu}} t}}{r^{2 \nu}+2 r^{\nu} \cos \nu \pi+1} d r, \quad \nu \in(0,1) .
$$

In a previous work (see Orsingher and Polito [15]) we proved a useful subordination representation for the fractional pure birth process (3.2). This can be viewed as a classical birth process stopped at a random time $T_{2 \nu}(t)$ possessing density function coinciding with the folded solution to the fractional diffusion equation

$$
\left\{\begin{array}{l}
\frac{\partial^{2 \nu} g}{\partial t^{2 \nu}}=\frac{\partial^{2} g}{\partial x^{2}}, \quad 0<\nu \leq 1 \\
g(x, 0)=\delta(x)
\end{array}\right.
$$

with the additional condition $g_{t}(x, 0)=0$ for $1 / 2<\nu \leq 1$. In other words $\mathcal{N}^{\nu}(t)=\mathcal{N}\left(T_{2 \nu}(t)\right), t>0$. It can be shown that $f_{T_{2 \nu}}(s, t)=\operatorname{Pr}\left\{T_{2 \nu}(t) \in d s\right\}$ is also a solution to

$$
\frac{\partial^{\nu} f}{\partial t^{\nu}}=-\frac{\partial f}{\partial s}
$$

(see Orsingher et al. [16]).

Theorem 3.1. The fractional nonlinear pure birth process is a renewal process with intermediate waiting times $T_{k}^{\nu}$ with law

$$
\operatorname{Pr}\left\{T_{k}^{\nu} \in d s\right\}=\lambda_{k} s^{\nu-1} E_{\nu, \nu}\left(-\lambda_{k} s^{\nu}\right) d s, \quad k \geq 1, s>0,
$$

where $T_{k}^{\nu}$ is the random time separating the $k$ th and $(k+1)$ th birth.

Proof. We prove this result by induction. Denoting $Z_{k}^{\nu}=T_{1}^{\nu}+\cdots+T_{k}^{\nu}$, we can certainly write that

$$
\operatorname{Pr}\left\{T_{1}^{\nu}+\cdots+T_{k}^{\nu} \in d t\right\}=\int_{0}^{t} \operatorname{Pr}\left\{T_{k}^{\nu} \in d(t-s)\right\} \operatorname{Pr}\left\{T_{1}^{\nu}+\cdots+T_{k-1}^{\nu} \in d s\right\},
$$


where $\operatorname{Pr}\left\{T_{1}^{\nu}+\cdots+T_{k-1}^{\nu} \in d s\right\} / d s=\frac{d}{d s} \operatorname{Pr}\left\{\mathcal{N}^{\nu}(s) \geq k\right\}$. By resorting to Laplace transforms, from (3.9), we obtain that

$$
\begin{aligned}
\int_{0}^{\infty} e^{-\mu t} \operatorname{Pr}\left\{T_{1}^{\nu}+\cdots+T_{k}^{\nu} \in d t\right\} & =\int_{0}^{\infty} e^{-\mu t} d t \int_{0}^{t} \operatorname{Pr}\left\{T_{k}^{\nu} \in d(t-s)\right\} \operatorname{Pr}\left\{T_{1}^{\nu}+\cdots+T_{k-1}^{\nu} \in d s\right\} \\
& =\int_{0}^{\infty} \operatorname{Pr}\left\{T_{1}^{\nu}+\cdots+T_{k-1}^{\nu} \in d s\right\} \int_{s}^{\infty} e^{-\mu t} \operatorname{Pr}\left\{T_{k}^{\nu} \in d(t-s)\right\} \\
& =\int_{0}^{\infty} e^{-\mu s} \operatorname{Pr}\left\{T_{1}^{\nu}+\cdots+T_{k-1}^{\nu} \in d s\right\} \int_{0}^{\infty} e^{-\mu y} \operatorname{Pr}\left\{T_{k}^{\nu} \in d y\right\} \\
& =\prod_{j=1}^{k} \int_{0}^{\infty} e^{-\mu s} \operatorname{Pr}\left\{T_{j}^{\nu} \in d s\right\}=\prod_{j=1}^{k} \frac{\lambda_{j}}{\mu^{\nu}+\lambda_{j}} .
\end{aligned}
$$

We observe that

$$
\operatorname{Pr}\left\{T_{1}^{\nu} \in d s\right\} / d s=\frac{d}{d s} \operatorname{Pr}\left\{\mathcal{N}^{\nu}(s) \geq 2\right\}=-\frac{d}{d s} E_{\nu, 1}\left(-\lambda_{1} s^{\nu}\right)=\lambda_{1} s^{\nu-1} E_{\nu, \nu}\left(-\lambda_{1} s^{\nu}\right),
$$

and that

$$
\begin{aligned}
\operatorname{Pr}\left\{T_{1}^{\nu}+T_{2}^{\nu} \in d s\right\} & =\frac{d}{d s}\left[1-\operatorname{Pr}\left\{\mathcal{N}^{\nu}(s)=1\right\}-\operatorname{Pr}\left\{\mathcal{N}^{\nu}(s)=2\right\}\right] \\
& =\lambda_{1} s^{\nu-1} E_{\nu, \nu}\left(-\lambda_{1} s^{\nu}\right)+\lambda_{1}\left[\lambda_{1} \frac{E_{\nu, \nu}\left(-\lambda_{1} s^{\nu}\right)}{\lambda_{2}-\lambda_{1}}+\lambda_{2} \frac{E_{\nu, \nu}\left(-\lambda_{2} s^{\nu}\right)}{\lambda_{1}-\lambda_{2}}\right] s^{\nu-1} \\
& =\frac{\lambda_{1} \lambda_{2}}{\lambda_{2}-\lambda_{1}} s^{\nu-1}\left[E_{\nu, \nu}\left(-\lambda_{1} s^{\nu}\right)-E_{\nu, \nu}\left(-\lambda_{2} s^{\nu}\right)\right]
\end{aligned}
$$

For $k=2$, relation (3.10) simplifies to

$$
\int_{0}^{\infty} e^{-\mu t} \operatorname{Pr}\left\{T_{1}^{\nu}+T_{2}^{\nu} \in d t\right\}=\frac{\lambda_{1}}{\mu^{\nu}+\lambda_{1}} \frac{\lambda_{2}}{\mu^{\nu}+\lambda_{2}},
$$

and this coincides with the Laplace transform of (3.12).

Theorem 3.2. The mean value $\mathbb{E} \mathcal{N}^{\nu}(t)$, for the fractional nonlinear pure birth process has the form:

$$
\mathbb{E} \mathcal{N}^{\nu}(t)=1+\sum_{k=1}^{\infty}\left\{1-\sum_{m=1}^{k} \prod_{l=1, l \neq m}^{k} \frac{\lambda_{l}}{\lambda_{l}-\lambda_{m}} E_{\nu, 1}\left(-\lambda_{m} t^{\nu}\right)\right\} .
$$

Proof. In light of the subordination relation $\mathcal{N}^{\nu}(t)=\mathcal{N}\left(T_{2 \nu}(t)\right)$, and of result (2.4), we can write that

$$
\begin{aligned}
\mathbb{E} \mathcal{N}^{\nu}(t) & =\int_{0}^{\infty} \mathbb{E} \mathcal{N}(s) \operatorname{Pr}\left\{T_{2 \nu}(t) \in d s\right\} \\
& =1+\sum_{k=1}^{\infty}\left\{1-\sum_{m=1}^{k} \prod_{l=1, l \neq m}^{k} \frac{\lambda_{l}}{\lambda_{l}-\lambda_{m}} \int_{0}^{\infty} e^{-\lambda_{m} s} \operatorname{Pr}\left\{T_{2 \nu}(t) \in d s\right\}\right\} \\
& =1+\sum_{k=1}^{\infty}\left\{1-\sum_{m=1}^{k} \prod_{l=1, l \neq m}^{k} \frac{\lambda_{l}}{\lambda_{l}-\lambda_{m}} E_{\nu, 1}\left(-\lambda_{m} t^{\nu}\right)\right\} .
\end{aligned}
$$


In the previous steps we assumed that

$$
\int_{0}^{\infty} e^{-\lambda_{m} s} f_{T_{2 \nu}}(t, s) d s=\int_{0}^{\infty} e^{-\lambda_{m} s} \operatorname{Pr}\left\{T_{2 \nu}(t) \in d s\right\}=E_{\nu, 1}\left(-\lambda_{m} t^{\nu}\right) .
$$

We give here some details of this result. The density $f_{T_{2 \nu}}(z, s), z>0, s>0$, is obtained by folding the solution of the fractional diffusion equation

$$
\frac{\partial^{2 \nu} u}{\partial z^{2 \nu}}=\frac{\partial^{2} u}{\partial s^{2}}
$$

which reads

$$
u(z, s)=\frac{1}{z^{\nu}} W_{-\nu, 1-\nu}\left(-\frac{s}{z^{\nu}}\right), \quad s>0, z>0,
$$

where $W_{-\nu, 1-\nu}(-\xi)$ is a Wright function defined as

$$
W_{-\nu, 1-\nu}(-\xi)=\sum_{r=0}^{\infty} \frac{(-\xi)^{r}}{r ! \Gamma(1-\nu(r+1))}
$$

Therefore

$$
\begin{aligned}
\frac{1}{z^{\nu}} \int_{0}^{\infty} e^{-\lambda_{m} s} W_{-\nu, 1-\nu}\left(-\frac{s}{z^{\nu}}\right) d s & =\frac{1}{z^{\nu}} \int_{0}^{\infty} e^{-\lambda_{m} s} \sum_{k=0}^{\infty}\left(-\frac{s}{z^{\nu}}\right)^{k} \frac{1}{k ! \Gamma(-\nu k+1-\nu)} d s \\
& =\frac{1}{z^{\nu}} \sum_{k=0}^{\infty} \frac{(-1)^{k}}{\lambda_{m}^{k+1} \Gamma(-\nu k+1-\nu)} \frac{1}{\left(z^{\nu}\right)^{k}}=\frac{1}{\lambda_{m} z^{\nu}} E_{-\nu, 1-\nu}\left(-\frac{1}{\lambda_{m} z^{\nu}}\right)
\end{aligned}
$$

(by formula (5.1) page 1825, Beghin and Orsingher [3])

$$
=E_{\nu, 1}\left(-\lambda_{m} z^{\nu}\right) \text {. }
$$

Remark 3.1. We can extract, from (3.14), the mean value of the fractional linear birth process obtained in Orsingher and Polito [15], formula (3.42), as follows. By considering that $\lambda_{m}=\lambda \cdot m$, formula (3.14) becomes

$$
\begin{aligned}
\mathbb{E} N^{\nu}(t) & =1+\sum_{k=1}^{\infty}\left\{1-\sum_{m=1}^{k}(-1)^{m-1} \frac{k !}{m !(k-m) !} E_{\nu, 1}\left(-\lambda m t^{\nu}\right)\right\} \\
& =1+\sum_{k=1}^{\infty}\left\{1+\sum_{m=1}^{k}(-1)^{m}\left(\begin{array}{c}
k \\
m
\end{array}\right) E_{\nu, 1}\left(-\lambda m t^{\nu}\right)\right\} \\
& =1+\sum_{k=1}^{\infty} \sum_{m=0}^{k}\left(\begin{array}{c}
k \\
m
\end{array}\right)(-1)^{m} E_{\nu, 1}\left(-\lambda m t^{\nu}\right)=\sum_{k=0}^{\infty} \sum_{m=0}^{k}\left(\begin{array}{c}
k \\
m
\end{array}\right)(-1)^{m} E_{\nu, 1}\left(-\lambda m t^{\nu}\right) .
\end{aligned}
$$

In order to obtain the desired result we pass to Laplace transforms and extract from (3.21) that

$$
\begin{aligned}
\int_{0}^{\infty} e^{-\mu t} \mathbb{E} N^{\nu}(t) d t & =\sum_{k=0}^{\infty} \sum_{m=0}^{k}\left(\begin{array}{c}
k \\
m
\end{array}\right)(-1)^{k} \int_{0}^{\infty} e^{-\mu t} E_{\nu, 1}\left(-\lambda m t^{\nu}\right) d t \\
& =\sum_{k=0}^{\infty} \sum_{m=0}^{k}\left(\begin{array}{c}
k \\
m
\end{array}\right)(-1)^{k} \frac{\mu^{\nu-1}}{\mu^{\nu}+\lambda m}=\mu^{\nu-1} \int_{0}^{\infty} \sum_{k=0}^{\infty} \sum_{m=0}^{k}\left(\begin{array}{c}
k \\
m
\end{array}\right)(-1)^{k} e^{-w \mu^{\nu}} e^{-w \lambda m} d w
\end{aligned}
$$




$$
\begin{aligned}
& =\mu^{\nu-1} \int_{0}^{\infty} \sum_{k=0}^{\infty} e^{-w \mu^{\nu}}\left(1-e^{-w \lambda}\right)^{k} d w=\mu^{\nu-1} \int_{0}^{\infty} \frac{e^{-w \mu^{\nu}}}{1-\left(1-e^{-w \lambda}\right)} d w \\
& =\mu^{\nu-1} \int_{0}^{\infty} e^{-w \mu^{\nu}+w \lambda} d w=\frac{\mu^{\nu-1}}{\mu^{\nu}-\lambda} .
\end{aligned}
$$

By inverting the Laplace transform above, we can conclude that

$$
\mathbb{E} N^{\nu}(t)=E_{\nu, 1}\left(\lambda t^{\nu}\right),
$$

thus confirming our previous result.

Here we remark that another interpretation in terms of random birth rates can be highlighted. If we write

$$
\begin{aligned}
p_{k}^{\nu}(t) & =\operatorname{Pr}\left\{\mathcal{N}^{\nu}(t)=k \mid \mathcal{N}^{\nu}(0)=1\right\}=\int_{0}^{\infty} p_{k}(s) \operatorname{Pr}\left\{T_{2 \nu}(t) \in d s\right\} \\
& =\prod_{j=1}^{k-1} \lambda_{j} \sum_{m=1}^{k} \frac{1}{\prod_{l=1, l \neq m}^{k}\left(\lambda_{l}-\lambda_{m}\right)} \int_{0}^{\infty} e^{-\lambda_{m} s} \operatorname{Pr}\left\{T_{2 \nu}(t) \in d s\right\} \\
& =\prod_{j=1}^{k-1} \lambda_{j} \sum_{m=1}^{k} \frac{1}{\prod_{l=1, l \neq m}^{k}\left(\lambda_{l}-\lambda_{m}\right)} \int_{0}^{\infty} e^{-\lambda_{m} s} \frac{1}{t^{\nu}} W_{-\nu, 1-\nu}\left(-\frac{s}{t^{\nu}}\right) d s \\
& =\prod_{j=1}^{k-1} \lambda_{j} \sum_{m=1}^{k} \frac{1}{\prod_{l=1, l \neq m}^{k}\left(\lambda_{l}-\lambda_{m}\right)} \int_{0}^{\infty} e^{-\lambda_{m} \xi t^{\nu}} W_{-\nu, 1-\nu}(-\xi) d \xi \\
& =\int_{0}^{\infty} W_{-\nu, 1-\nu}(-\xi) \operatorname{Pr}\left\{\mathcal{N}_{\xi}\left(t^{\nu}\right)=k \mid \mathcal{N}_{\xi}(0)=1\right\} d \xi,
\end{aligned}
$$

we have that a fractional nonlinear pure birth process can be considered as a classical nonlinear pure birth process evaluated at a rescaled time $t^{\nu}$ and with random rates $\lambda_{k} \Xi, k \geq 1$, where $\Xi$ is a random variable with density function

$$
f_{\Xi}(\xi)=W_{-\nu, 1-\nu}(-\xi), \quad \xi \in \mathbb{R}^{+} .
$$

From (3.24), the following interpretation also holds:

$$
\mathcal{N}^{\nu}(t) \stackrel{\text { i.d. }}{=} \mathcal{N}\left(\Xi t^{\nu}\right) .
$$

Note also that, from (3.5) and (3.24), we have that

$$
E_{\nu, 1}\left(-z t^{\nu}\right)=\int_{0}^{\infty} e^{-r z t^{\nu}} W_{-\nu, 1-\nu}(-r) d r=\frac{\sin \nu \pi}{\pi} \int_{0}^{\infty} e^{-r z^{\frac{1}{\nu}}} \frac{r^{\nu-1}}{r^{2 \nu}+2 r^{\nu} \cos \nu \pi+1} d r
$$

which illustrates an interesting relation between the Wright function and the law of $\mathcal{W}_{1}$ (see (1.21)). Equation (3.27) can be derived directly as follows.

$$
\begin{aligned}
& \int_{0}^{\infty} e^{-\gamma x} \frac{1}{\lambda t^{\nu}} W_{-\nu, 1-\nu}\left(-\frac{x}{\lambda t^{\nu}}\right) d x=\frac{1}{\lambda t^{\nu}} \sum_{m=0}^{\infty} \frac{(-1)^{m}}{m ! \Gamma\left(-\frac{\nu}{m}+1-\nu\right)} \frac{1}{\left(\lambda t^{\nu}\right)^{m}} \int_{0}^{\infty} e^{-\gamma x} x^{m} d x \\
& =\frac{1}{\lambda t^{\nu}} \sum_{m=0}^{\infty} \frac{(-1)^{m}}{\Gamma\left(-\frac{\nu}{m}+1-\nu\right)} \frac{1}{\gamma\left(\gamma \lambda t^{\nu}\right)^{m}}=\frac{1}{\gamma \lambda t^{\nu}} E_{-\nu, 1-\nu}\left(-\frac{1}{\gamma \lambda t^{\nu}}\right)
\end{aligned}
$$

(by formula (5.1), page 1825, Beghin and Orsingher [3] ) 


$$
=E_{\nu, 1}\left(-\gamma \lambda t^{\nu}\right)=\frac{\sin \nu \pi}{\pi} \int_{0}^{\infty} \frac{e^{-(\gamma \lambda)^{\frac{1}{\nu}} t r} r^{\nu-1}}{\left(r^{\nu}+\cos \nu \pi\right)^{2}+\sin ^{2} \nu \pi} d r .
$$

This yields (3.27) for $z=\gamma \lambda$.

For further details on fractional pure birth process the reader can refer to Orsingher and Polito [15] while Mittag-Leffler functions are extensively analysed in Kilbas et al. [9] .

\subsection{Fractional pure birth process stopped at $T_{t}$}

We consider here the composition of a fractional nonlinear pure birth process, denoted as $\mathcal{N}^{\nu}(t), t>0$, $\nu \in(0,1]$ with the first-passage time $T_{t}$ of a standard Brownian motion. In the following theorem we derive an interesting integral representation for the state probabilities $\hat{p}_{k}^{\nu}(t), t>0, k \geq 1$, of $\hat{\mathcal{N}}^{\nu}(t)=\mathcal{N}^{\nu}\left(T_{t}\right), t>0$, $\nu \in(0,1)$.

Theorem 3.3. Let $\mathcal{N}^{\nu}(t), t>0, \nu \in(0,1)$, be a fractional nonlinear pure birth process and $T_{t}$ be the first-passage time process of the standard Brownian motion with distribution $q(t, s)$. The state probabilities $\hat{p}_{k}^{\nu}(t)=\operatorname{Pr}\left\{\mathcal{N}^{\nu}\left(T_{t}\right)=k \mid \mathcal{N}^{\nu}(0)=1\right\}$ possess the following integral form

$$
\hat{p}_{k}^{\nu}(t)= \begin{cases}\prod_{j=1}^{k-1} \lambda_{j} \sum_{m=1}^{k} \frac{1}{\prod_{l=1, l \neq m}^{k}\left(\lambda_{l}-\lambda_{m}\right)} \frac{1}{i \pi} \int_{0}^{\infty} \frac{E_{2 \nu, 1}\left(-x^{2 \nu} e^{i \pi \nu}\right)-E_{2 \nu, 1}\left(-x^{2 \nu} e^{-i \pi \nu}\right)}{x+t \sqrt{2 \lambda_{m}^{\frac{1}{\nu}}}} d x, & k>1, \\ \frac{1}{i \pi} \int_{0}^{\infty} \frac{E_{2 \nu, 1}\left(-x^{2 \nu} e^{i \pi \nu}\right)-E_{2 \nu, 1}\left(-x^{2 \nu} e^{-i \pi \nu}\right)}{x+t \sqrt{2 \lambda_{1}^{\frac{1}{\nu}}}} d x, & k=1 .\end{cases}
$$

Proof. It is sufficient to prove (3.29) in the case $k>1$, since the case $k=1$ is analogous. We have

$$
\hat{p}_{k}^{\nu}(t)=\int_{0}^{\infty} p_{k}^{\nu}(s) q(t, s) d s=\prod_{j=1}^{k-1} \lambda_{j} \sum_{m=1}^{k} \frac{1}{\prod_{l=1, l \neq m}^{k}\left(\lambda_{l}-\lambda_{m}\right)} \int_{0}^{\infty} E_{\nu, 1}\left(-\lambda_{m} s^{\nu}\right) \frac{t e^{-\frac{t^{2}}{2 s}}}{\sqrt{2 \pi s^{3}}} d s .
$$

In order to prove (3.29), by taking into consideration formula (3.5), we do the following calculations

$$
\begin{aligned}
& \int_{0}^{\infty} E_{\nu, 1}\left(-\lambda_{m} s^{\nu}\right) \frac{t e^{-\frac{t^{2}}{2 s}}}{\sqrt{2 \pi s^{3}}} d s=\int_{0}^{\infty} \frac{t e^{-\frac{t^{2}}{2 s}}}{\sqrt{2 \pi s^{3}}} \int_{0}^{\infty} \frac{\sin \nu \pi}{\pi} \frac{r^{\nu-1} e^{-r \lambda_{m}^{\frac{1}{\nu}} s}}{r^{2 \nu}+2 r^{\nu} \cos \nu \pi+1} d r d s \\
& =\frac{\sin \nu \pi}{\pi} \int_{0}^{\infty} \frac{r^{\nu-1}}{r^{2 \nu}+2 r^{\nu} \cos \nu \pi+1} \int_{0}^{\infty} e^{-r \lambda_{m}^{\frac{1}{\nu}}} \frac{t e^{-\frac{t^{2}}{2 s}}}{\sqrt{2 \pi s^{3}}} d s d r \\
& =\frac{\sin \nu \pi}{\pi} \int_{0}^{\infty} \frac{r^{\nu-1}}{r^{2 \nu}+2 r^{\nu} \cos \nu \pi+1} e^{-t \sqrt{2 r \lambda_{m}^{\frac{1}{\nu}}}} d r=\frac{\sin \nu \pi}{\pi} \int_{0}^{\infty} \frac{r^{\nu-1} e^{-t} \sqrt{2 r \lambda_{m}^{\frac{1}{\nu}}}}{\left(r^{\nu}+e^{i \pi \nu}\right)\left(r^{\nu}+e^{-i \pi \nu}\right)} d r \\
& =\frac{1}{2 i \pi} \int_{0}^{\infty}\left(\frac{1}{r^{\nu}+e^{i \pi \nu}}-\frac{1}{r^{\nu}+e^{-i \pi \nu}}\right) r^{\nu-1} e^{-t \sqrt{2 \lambda_{m}^{\frac{1}{\nu}}}} d r \\
& =\frac{1}{i \pi} \int_{0}^{\infty}\left(\frac{1}{w^{2 \nu}+e^{i \pi \nu}}-\frac{1}{w^{2 \nu}+e^{-i \pi \nu}}\right) w^{2 \nu-1} e^{-t w \sqrt{2 \lambda_{m}^{\frac{1}{\nu}}}} d w \\
& =\frac{1}{i \pi} \int_{0}^{\infty}\left[\frac{w^{2 \nu-1}}{w^{2 \nu}+e^{i \pi \nu}}-\frac{w^{2 \nu-1}}{w^{2 \nu}+e^{-i \pi \nu}}\right] e^{-t w \sqrt{2 \lambda_{m}^{\frac{1}{\nu}}}} d w .
\end{aligned}
$$

By using the Laplace transform (3.4) we obtain

$$
\int_{0}^{\infty} E_{\nu, 1}\left(-\lambda_{m} s^{\nu}\right) \frac{t e^{-\frac{t^{2}}{2 s}}}{\sqrt{2 \pi s^{3}}} d s
$$




$$
\begin{aligned}
& =\frac{1}{i \pi} \int_{0}^{\infty}\left[\int_{0}^{\infty} e^{-w x} E_{2 \nu, 1}\left(-x^{2 \nu} e^{i \pi \nu}\right) d x-\int_{0}^{\infty} e^{-w x} E_{2 \nu, 1}\left(-x^{2 \nu} e^{-i \pi \nu}\right) d x\right] e^{-t w \sqrt{2 \lambda_{m}^{\frac{1}{\nu}}}} d w \\
& =\frac{1}{i \pi} \int_{0}^{\infty} \frac{E_{2 \nu, 1}\left(-x^{2 \nu} e^{i \pi \nu}\right)-E_{2 \nu, 1}\left(-x^{2 \nu} e^{-i \pi \nu}\right)}{x+t \sqrt{2 \lambda_{m}^{\frac{1}{\nu}}}} d x .
\end{aligned}
$$

Formula (3.29) is then proved by combining (3.30) and (3.32).

Remark 3.2. If $\lambda_{k}=k \lambda, k \geq 1$, the state probabilities $\hat{p}_{k}^{\nu}(t)=\operatorname{Pr}\left\{N^{\nu}\left(T_{t}\right)=k \mid N^{\nu}(0)=1\right\}$ of a fractional linear pure birth process stopped at $T_{t}$ read

$$
\hat{p}_{k}^{\nu}(t)= \begin{cases}\sum_{m=1}^{k}\left(\begin{array}{c}
k-1 \\
m-1
\end{array}\right)(-1)^{m-1} \frac{1}{i \pi} \int_{0}^{\infty} \frac{E_{2 \nu, 1}\left(-x^{2 \nu} e^{i \pi \nu}\right)-E_{2 \nu, 1}\left(-x^{2 \nu} e^{-i \pi \nu}\right)}{x+t \sqrt{2 \lambda^{\frac{1}{\nu}} m}} d x, & k>1, \\
\frac{1}{i \pi} \int_{0}^{\infty} \frac{E_{2 \nu, 1}\left(-x^{2 \nu} e^{i \pi \nu}\right)-E_{2 \nu, 1}\left(-x^{2 \nu} e^{-i \pi \nu}\right)}{x+t \sqrt{2 \lambda^{\frac{1}{\nu}}}} d x, & k=1 .\end{cases}
$$

This result can be obtained by means of methods similar to those of Theorem 3.3 .

Remark 3.3. By considering formula (3.30) and the representation (3.5) we can give an interesting interpretation of the process $\hat{\mathfrak{N}}^{1 / 2}(t)=\mathfrak{N}^{1 / 2}\left(T_{t}\right), t>0$, as follows (again, we treat the case $k \geq 1$ since the case $k=1$ is analogous)

$$
\begin{aligned}
\hat{p}_{k}^{\nu}(t) & =\int_{0}^{\infty} p_{k}^{\nu}(s) q(t, s) d s \\
& =\prod_{j=1}^{k-1} \lambda_{j} \sum_{m=1}^{k} \frac{1}{\prod_{l=1, l \neq m}^{k}\left(\lambda_{l}-\lambda_{m}\right)} \int_{0}^{\infty} E_{\nu, 1}\left(-\lambda_{m} s^{\nu}\right) \frac{t e^{-\frac{t^{2}}{2 s}}}{\sqrt{2 \pi s^{3}}} d s \\
& =\prod_{j=1}^{k-1} \lambda_{j} \sum_{m=1}^{k} \frac{1}{\prod_{l=1, l \neq m}^{k}\left(\lambda_{l}-\lambda_{m}\right)} \frac{\sin \nu \pi}{\pi} \int_{0}^{\infty} \frac{r^{\nu-1}}{r^{2 \nu}+2 r^{\nu} \cos \nu \pi+1} e^{-t \sqrt{2 r \lambda_{m}^{\frac{1}{\nu}}}} d r \\
& =\frac{\sin \nu \pi}{\pi} \int_{0}^{\infty} \frac{r^{\nu-1}}{r^{2 \nu}+2 r^{\nu} \cos \nu \pi+1} \prod_{j=1}^{k-1} \lambda_{j} \sum_{m=1}^{k} \frac{1}{\prod_{l=1, l \neq m}^{k}\left(\lambda_{l}-\lambda_{m}\right)} e^{2 r \lambda_{m}^{\frac{1}{\nu}}} d r .
\end{aligned}
$$

If $\nu=1 / 2$ we obtain the following expression

$$
\begin{aligned}
\hat{p}_{k}^{\frac{1}{2}}(t) & =\frac{1}{\pi} \int_{0}^{\infty} \frac{1}{\sqrt{r}(r+1)} \prod_{j=1}^{k-1}\left(\sqrt{2 r} \lambda_{j}\right) \sum_{m=1}^{k} \frac{1}{\prod_{l=1, l \neq m}^{k}\left(\sqrt{2 r} \lambda_{l}-\sqrt{2 r} \lambda_{m}\right)} e^{-t \sqrt{2 r} \lambda_{m}} d r \\
& =\frac{\sqrt{2}}{\pi} \int_{0}^{\infty} \frac{1}{\left(\frac{w^{2}}{2}+1\right)} \operatorname{Pr}\left\{\mathcal{N}_{w}(t)=k \mid \mathcal{N}_{w}(0)=1\right\} d w
\end{aligned}
$$

where $\mathfrak{N}_{W}(t), t>0$, is a classical nonlinear birth process (2.1) with random birth rates $\left(W \lambda_{k}\right), k \geq 1$ where $W$ is a folded Cauchy r.v. with p.d.f.

$$
f_{W}(w)=\frac{\sqrt{2}}{\pi\left(\frac{w^{2}}{2}+1\right)}, \quad w \in \mathbb{R}^{+} .
$$

It is possible to highlight a further interpretation by rewriting formula (3.35) in the following way

$$
\hat{p}_{k}^{\frac{1}{2}}(t)=\frac{\sqrt{2}}{\pi} \int_{0}^{\infty} \frac{1}{t\left(\frac{s^{2}}{2 t^{2}}+1\right)} \prod_{j=1}^{k-1} \lambda_{j} \sum_{m=1}^{k} \frac{1}{\prod_{l=1, l \neq m}^{k}\left(\lambda_{l}-\lambda_{m}\right)} e^{-s \lambda_{m}} d s
$$




$$
=\int_{0}^{\infty} \operatorname{Pr}\{\mathcal{N}(s)=k\} \operatorname{Pr}\{|C(\sqrt{2} t)| \in d s\}
$$

where $C(\sqrt{2} t), t>0$ is a Cauchy process with rescaled time, possessing transition density

$$
f_{C}(t, s)=\frac{1}{\pi} \frac{\sqrt{2} t}{s^{2}+(\sqrt{2} t)^{2}}, \quad t>0, s \in \mathbb{R} .
$$

The process $\hat{\mathcal{N}}^{1 / 2}(t)=\mathfrak{N}^{1 / 2}\left(T_{t}\right)$ can thus be written as $\hat{\mathfrak{N}}^{1 / 2}(t)=\mathcal{N}(|C(\sqrt{2} t)|)$.

\subsubsection{Iterated compositions}

In the next theorem we present the explicit form of the state probabilities $\tilde{p}_{k}^{\nu}(t), t>0, k \geq 1$, for the process

$$
\tilde{\mathcal{N}}^{\nu}(t)=\mathfrak{N}^{\nu}\left[T_{T_{\cdot \cdot T_{t}^{n}}^{2}}^{1}\right], \quad t>0, \nu \in(0,1] .
$$

and in the following remark an interesting interpretation for that process when $\nu=1 / 2^{n}, n \in \mathbb{N}$, is given.

Theorem 3.4. Let $\mathcal{N}^{\nu}(t), t>0, \nu \in(0,1]$, be a fractional nonlinear pure birth process and let $T_{t}^{1}, T_{t}^{2}$, $\ldots T_{t}^{n}, n \in \mathbb{N}$, be $n$ independent first-passage time processes at $t$ of the standard Brownian motion. The process

$$
\tilde{\mathcal{N}}^{\nu}(t)=\mathcal{N}^{\nu}\left[T_{T^{2} \cdot T_{t}^{n}}^{1}\right], \quad t>0, \nu \in(0,1]
$$

has the following distribution

$$
\tilde{p}_{k}^{\nu}(t)= \begin{cases}\prod_{j=1}^{k-1} \lambda_{j} \sum_{m=1}^{k} \frac{1}{\prod_{l=1, l \neq m}^{k}\left(\lambda_{l}-\lambda_{m}\right)} \frac{\sin \nu \pi}{\pi} \int_{0}^{\infty} \frac{r^{\nu-1} e^{-t r} \frac{1}{2^{n}} \lambda_{m}^{\frac{1}{2^{2 n}}}{ }_{2}\left(1-\frac{1}{2^{n}}\right)}{r^{2 \nu}+2 r^{\nu} \cos \nu \pi+1} d r, & k>1, t>0, \\ \frac{\sin \nu \pi}{\pi} \int_{0}^{\infty} \frac{r^{\nu-1} e^{-t \lambda_{1}^{2 n}} 2^{2}\left(1-\frac{1}{2^{n}}\right)}{r^{2 \nu}+2 r^{\nu} \cos \nu \pi+1} d r, & k=1, t>0 .\end{cases}
$$

Proof. We start by proving the case $n=2$ since the case $n=1$ is already proved in Theorem 3.3. We omit the details for the case $k=1$ and directly treat the case $k \geq 2$. We have

$$
\begin{aligned}
& \int_{0}^{\infty} \hat{p}_{k}^{\nu}(s) q(t, s) d s \\
& =\prod_{j=1}^{k-1} \lambda_{j} \sum_{m=1}^{k} \frac{1}{\prod_{l=1, l \neq m}^{k}\left(\lambda_{l}-\lambda_{m}\right)} \frac{\sin \nu \pi}{\pi} \int_{0}^{\infty} \frac{r^{\nu-1}}{r^{2 \nu}+2 r^{\nu} \cos \nu \pi+1} \int_{0}^{\infty} e^{-s(2 r)^{\frac{1}{2}} \lambda_{m}^{\frac{1}{2 \nu}}} \frac{t e^{-\frac{t^{2}}{2 s}}}{\sqrt{2 \pi s^{3}}} d s d r \\
& =\prod_{j=1}^{k-1} \lambda_{j} \sum_{m=1}^{k} \frac{1}{\prod_{l=1, l \neq m}^{k}\left(\lambda_{l}-\lambda_{m}\right)} \frac{\sin \nu \pi}{\pi} \int_{0}^{\infty} \frac{r^{\nu-1}}{r^{2 \nu}+2 r^{\nu} \cos \nu \pi+1} e^{-t \sqrt{2 r \lambda_{m}^{\frac{1}{\nu}}}} d r .
\end{aligned}
$$

It is now straightforward to generalise formula (3.42) for $n$ compositions, as follows

$$
\begin{aligned}
\tilde{p}_{k}^{\nu}(t) & =\prod_{j=1}^{k-1} \lambda_{j} \sum_{m=1}^{k} \frac{1}{\prod_{l=1, l \neq m}^{k}\left(\lambda_{l}-\lambda_{m}\right)} \frac{\sin \nu \pi}{\pi} \int_{0}^{\infty} \frac{r^{\nu-1}}{r^{2 \nu}+2 r^{\nu} \cos \nu \pi+1} e^{-t r^{\frac{1}{2^{n}}} \lambda_{m}^{\frac{1}{\nu^{2 \pi}}} 2^{\sum_{i=1}^{n} \frac{1}{2^{i}}}} d r \\
& =\prod_{j=1}^{k-1} \lambda_{j} \sum_{m=1}^{k} \frac{1}{\prod_{l=1, l \neq m}^{k}\left(\lambda_{l}-\lambda_{m}\right)} \frac{\sin \nu \pi}{\pi} \int_{0}^{\infty} \frac{r^{\nu-1}}{r^{2 \nu}+2 r^{\nu} \cos \nu \pi+1} e^{-t r \frac{1}{2^{n}} \lambda_{m}^{\frac{1}{2^{2 \pi}}} 2^{\left(1-\frac{1}{2^{n}}\right)}} d r .
\end{aligned}
$$


Remark 3.4. Analogously to Remark 3.3, for $\nu=1 / 2^{n}, n \in \mathbb{N}$, it is possible to interpret formula (3.41) as follows

$$
\begin{aligned}
& \tilde{p}_{k}^{\frac{1}{2^{n}}}(t)=\prod_{j=1}^{k-1} \lambda_{j} \sum_{m=1}^{k} \frac{1}{\prod_{l=1, l \neq m}^{k}\left(\lambda_{l}-\lambda_{m}\right)} \frac{\sin \frac{\pi}{2^{n}}}{\pi} \int_{0}^{\infty} \frac{r^{\frac{1}{2^{n}}-1}}{r^{\frac{1}{2^{n-1}}+2 r^{\frac{1}{2^{n}}} \cos \frac{\pi}{2^{n}}+1} e^{-t r \frac{1}{2^{n}} 2^{\left(1-\frac{1}{2^{n}}\right)} \lambda_{m}} d r} \\
& \left(r=y^{2^{n}}\right) \\
& =\frac{\sin \frac{\pi}{2^{n}}}{\frac{\pi}{2^{n}}} \int_{0}^{\infty} \frac{d r}{r^{2}+2 r \cos \frac{\pi}{2^{n}}+1} \operatorname{Pr}\left\{\mathcal{N}\left(t r 2^{1-\frac{1}{2^{n}}}\right)=k\right\} .
\end{aligned}
$$

Therefore, the following representation holds

$$
\mathcal{N}^{\frac{1}{2^{n}}}\left[T_{T_{\cdot \cdot T_{t}^{n}}^{2}}^{1}\right]=\mathcal{N}\left(t \Omega 2^{1-\frac{1}{2^{n}}}\right),
$$

where $\Omega$ is a random variable with density

$$
f_{\Omega}(r)=\frac{\sin \frac{\pi}{2^{n}}}{\frac{\pi}{2^{n}}} \frac{1}{r^{2}+2 r \cos \frac{\pi}{2^{n}}+1}, \quad r \in \mathbb{R}^{+} .
$$

The density is a unimodal law which, for $n \rightarrow \infty$, becomes

$$
f(r)=\frac{1}{(1+r)^{2}}, \quad r \in \mathbb{R}^{+} .
$$

\subsection{Fractional pure birth process stopped at $\mathcal{S}^{\alpha}(t)$}

We consider the fractional nonlinear pure birth process stopped at a stable time $\mathcal{S}^{\alpha}(t)$ of order $0<\alpha \leq 1$ with Laplace transform

$$
\mathbb{E} e^{-z S^{\alpha}(t)}=\int_{0}^{\infty} e^{-z s} q_{\alpha}(t, s) d s=e^{-t z^{\alpha}},
$$

where $q_{\alpha}(t, s), s>0$, is the density of the stable process $\mathcal{S}^{\alpha}(t), t>0$.

We have that the probabilities

$$
\begin{aligned}
& \breve{p}_{k}^{\nu}(t)=\operatorname{Pr}\left\{\mathcal{N}^{\nu}\left(\mathcal{S}^{\alpha}(t)\right)=k \mid \mathcal{N}^{\nu}(0)=1\right\} \\
& =\prod_{j=1}^{k-1} \lambda_{j} \sum_{m=1}^{k} \frac{1}{\prod_{l=1, l \neq m}^{k}\left(\lambda_{l}-\lambda_{m}\right)} \int_{0}^{\infty} E_{\nu, 1}\left(-\lambda_{m} s^{\nu}\right) q_{\alpha}(t, s) d s \\
& =\prod_{j=1}^{k-1} \lambda_{j} \sum_{m=1}^{k} \frac{1}{\prod_{l=1, l \neq m}^{k}\left(\lambda_{l}-\lambda_{m}\right)} \frac{\sin \nu \pi}{\pi} \int_{0}^{\infty} \frac{r^{\nu-1}}{r^{2 \nu}+2 r^{\nu} \cos \nu \pi+1} \int_{0}^{\infty} e^{-r \lambda_{m}^{\frac{1}{\nu}} s} q_{\alpha}(t, s) d s d r \\
& =\prod_{j=1}^{k-1} \lambda_{j} \sum_{m=1}^{k} \frac{1}{\prod_{l=1, l \neq m}^{k}\left(\lambda_{l}-\lambda_{m}\right)} \frac{\sin \nu \pi}{\pi} \int_{0}^{\infty} \frac{r^{\nu-1}}{r^{2 \nu}+2 r^{\nu} \cos \nu \pi+1} e^{-t \lambda_{m}^{\frac{\alpha}{\nu}} r^{\alpha}} d r \\
& =\prod_{j=1}^{k-1} \lambda_{j} \sum_{m=1}^{k} \frac{1}{\prod_{l=1, l \neq m}^{k}\left(\lambda_{l}-\lambda_{m}\right)} \frac{\sin \nu \pi}{\alpha \pi} \int_{0}^{\infty} \frac{w^{\frac{\nu}{\alpha}-1}}{w^{2 \frac{\nu}{\alpha}}+2 w^{\frac{\nu}{\alpha}} \cos \nu \pi+1} e^{-t \lambda_{m}^{\frac{\alpha}{\nu}} w} d w \\
& =\prod_{j=1}^{k-1} \lambda_{j} \sum_{m=1}^{k} \frac{1}{\prod_{l=1, l \neq m}^{k}\left(\lambda_{l}-\lambda_{m}\right)} \mathbb{E} e^{-t \lambda_{m}^{\frac{\alpha}{\nu}} \mathcal{W}_{\alpha}},
\end{aligned}
$$


where $\mathcal{W}_{\alpha}$ is a random variable with density

$$
f_{\mathcal{W}_{\alpha}}(w)=\frac{\sin \nu \pi}{\alpha \pi} \frac{w^{\frac{\nu}{\alpha}-1}}{w^{2 \frac{\nu}{\alpha}}+2 w^{\frac{\nu}{\alpha}} \cos \nu \pi+1}, \quad w>0,0<\nu<1,
$$

(first obtained by Lamperti [10]). The density (3.50) coincides with the probability distribution of

$$
\mathcal{W}_{\alpha}=\left(\frac{S_{1}^{\nu}}{S_{2}^{\nu}}\right)^{\alpha}
$$

where $S_{1}^{\nu}, S_{2}^{\nu}$, are independent stable random variables with Laplace transform

$$
\mathbb{E} e^{-z S^{\nu}}=e^{-z^{\nu}}, \quad z>0,0<\nu<1 .
$$

If $\alpha=1, \mathbb{E} e^{-t \lambda_{m}^{\frac{1}{\nu}} \mathcal{W}_{\alpha}}=E_{\nu, 1}\left(-\lambda_{m} t^{\nu}\right)$ and (3.49) are the state probabilities of a fractional pure birth process, while for $\alpha=\nu, \mathbb{E} e^{-t \lambda_{m} \mathcal{W}_{\nu}}$ are the state probabilities of a pure birth process at time $t \mathcal{W}_{\nu}$ or, equivalently, a pure birth process at time $t$ with rates $\lambda_{k} \mathcal{W}_{\nu}$.

If we compare (3.49) with (2.55), we can conclude that the process

$$
\mathcal{N}^{\nu}\left(S^{\alpha}(t)\right)=\mathcal{N}\left(T_{2 \nu}\left(S^{\alpha}(t)\right)\right),
$$

can be represented as

$$
\mathcal{N}\left(\mathcal{S}^{\alpha / \nu}\left(t \mathcal{W}_{\alpha}\right)\right)
$$

if $0<\alpha<\nu<1$.

Remark 3.5. From formula (3.49), when $\alpha$ takes the form $\alpha=\nu / 2^{n}, n \in \mathbb{N}$, we have

$$
\breve{p}_{k}^{\nu}(t)=\frac{\sin \nu \pi}{\pi} \int_{0}^{\infty} \frac{r^{\nu-1}}{r^{2 \nu}+2 r^{\nu} \cos \nu \pi+1} \prod_{j=1}^{k-1} \lambda_{j} \sum_{m=1}^{k} \frac{e^{-t \lambda_{m}^{\frac{1}{2^{n}}} r^{\frac{\nu}{2^{n}}}}}{\prod_{l=1, l \neq m}^{k}\left(\lambda_{l}-\lambda_{m}\right)} d r .
$$

For $n \rightarrow \infty$, we obtain that $\breve{p}_{k}^{\nu}(t) \rightarrow 0, k>1$, and $\breve{p}_{1}^{\nu}(t) \rightarrow e^{-t}$. This shows that for $n \rightarrow \infty$, either the population istantaneously explodes or does not produce offsprings with exponential probability.

An alternative way of presenting the state probabilities (3.49) is based on the Mellin-Barnes representation of the Mittag-Leffler function

$$
E_{\nu, \mu}(x)=\frac{1}{2 \pi i} \int_{\gamma-i \infty}^{\gamma+i \infty} \frac{\Gamma(z) \Gamma(1-z)}{\Gamma(\mu-\nu z)} x^{-z} d z
$$

with $\nu>0, x \in \mathbb{C},|\arg (-x)|<\pi$ (see Kilbas et al. [9], page 44, formula (1.8.32)).

In view of (3.56), we can write (3.49) as follows

$$
\begin{aligned}
\breve{p}_{k}^{\nu}(t) & =\prod_{j=1}^{k-1} \lambda_{j} \sum_{m=1}^{k} \frac{1}{\prod_{l=1, l \neq m}^{k}\left(\lambda_{l}-\lambda_{m}\right)} \int_{0}^{\infty} \frac{1}{2 \pi i} \int_{\gamma-i \infty}^{\gamma+i \infty} \frac{\Gamma(z) \Gamma(1-z)}{\Gamma(1-\nu z)}\left(\lambda_{m} s^{\nu}\right)^{-z} d z q_{\alpha}(t, s) d s \\
& =\prod_{j=1}^{k-1} \lambda_{j} \sum_{m=1}^{k} \frac{1}{\prod_{l=1, l \neq m}^{k}\left(\lambda_{l}-\lambda_{m}\right)} \frac{1}{2 \pi i} \int_{\gamma-i \infty}^{\gamma+i \infty} \frac{\Gamma(z) \Gamma(1-z)}{\Gamma(1-\nu z)} \lambda_{m}^{-z} \int_{0}^{\infty} s^{-\nu z} q_{\alpha}(t, s) d s d z,
\end{aligned}
$$

where in the last member of (3.57) the Mellin transform of $q_{\alpha}(t, s)$ appears. 
The Mellin transform of the stable subordinator $\mathcal{S}^{\alpha}(t)$, with Laplace transform

$$
\mathbb{E} e^{-z S^{\alpha}(t)}=e^{-t z^{\alpha}}
$$

reads

$$
\mathbb{E}\left(\mathcal{S}^{\alpha}(t)\right)^{\eta-1}=\int_{0}^{\infty} s^{\eta-1} q_{\alpha}(t, s) d s=\frac{1}{\alpha} \Gamma\left(\frac{1-\eta}{\alpha}\right) \frac{1}{\Gamma(1-\eta)} t^{\frac{\eta-1}{\alpha}} .
$$

By inserting (3.59) into (3.57), we arrive at

$$
\begin{aligned}
\breve{p}_{k}^{\nu}(t) & =\prod_{j=1}^{k-1} \lambda_{j} \sum_{m=1}^{k} \frac{1}{\prod_{l=1, l \neq m}^{k}\left(\lambda_{l}-\lambda_{m}\right)} \frac{\alpha^{-1}}{2 \pi i} \int_{\gamma-i \infty}^{\gamma+i \infty} \frac{\Gamma(z) \Gamma(1-z) \Gamma\left(\frac{\nu}{\alpha} z\right)}{\Gamma(\nu z) \Gamma(1-\nu z)}\left(\lambda_{m} t^{\frac{\nu}{\alpha}}\right)^{-z} d z \\
& =\prod_{j=1}^{k-1} \lambda_{j} \sum_{m=1}^{k} \frac{1}{\prod_{l=1, l \neq m}^{k}\left(\lambda_{l}-\lambda_{m}\right)} \alpha^{-1} H_{2,3}^{2,1}\left[\begin{array}{cc}
\lambda_{m} t^{\frac{\nu}{\alpha}} & (0,1),(0, \nu) \\
(0,1),(0, \nu / \alpha),(0, \nu)
\end{array}\right] .
\end{aligned}
$$

We examine now in detail the case $\alpha=\nu$ in the next theorem.

Theorem 3.5. We have the following distributions:

1. $\operatorname{Pr}\left\{\mathcal{N}^{\nu}\left(\mathcal{S}^{\nu}(t)\right)=k\right\}=\operatorname{Pr}\left\{\mathcal{N}\left(T_{2 \nu}\left(\mathcal{S}^{\nu}(t)\right)\right)=k\right\}=\operatorname{Pr}\left\{\mathcal{N}\left(t \mathcal{W}_{\nu}\right)=k\right\}$,

2. $\operatorname{Pr}\left\{\mathcal{N}\left(\mathcal{S}^{\nu}\left(T_{2 \nu}(t)\right)\right)=k\right\}=\operatorname{Pr}\left\{\mathcal{N}\left(t \mathcal{W}_{1}\right)=k\right\}$,

for $k \geq 1, t>0$, where

$$
\mathcal{W}_{\alpha}=\left(\frac{S_{1}^{\nu}}{S_{2}^{\nu}}\right)^{\alpha}
$$

and has distribution (3.50).

Proof. For $k>1$ we can write that

$$
\begin{aligned}
\operatorname{Pr} & \left\{\mathcal{N}\left(T_{2 \nu}\left(\mathcal{S}^{\nu}(t)\right)\right)=k\right\} \\
= & \prod_{j=1}^{k-1} \lambda_{j} \sum_{m=1}^{k} \frac{1}{\prod_{l=1, l \neq m}^{k}\left(\lambda_{l}-\lambda_{m}\right)} \int_{0}^{\infty} e^{-\lambda_{m} s} \operatorname{Pr}\left\{T_{2 \nu}\left(\mathcal{S}^{\nu}(t)\right) \in d s\right\} \\
= & \prod_{j=1}^{k-1} \lambda_{j} \sum_{m=1}^{k} \frac{1}{\prod_{l=1, l \neq m}^{k}\left(\lambda_{l}-\lambda_{m}\right)} \int_{0}^{\infty} e^{-\lambda_{m} s} \int_{0}^{\infty} f_{T_{2 \nu}}(z, s) q_{\nu}(t, z) d z d s \\
= & \prod_{j=1}^{k-1} \lambda_{j} \sum_{m=1}^{k} \frac{1}{\prod_{l=1, l \neq m}^{k}\left(\lambda_{l}-\lambda_{m}\right)} \int_{0}^{\infty} E_{\nu, 1}\left(-\lambda_{m} z^{\nu}\right) q_{\nu}(t, z) d z \\
= & \prod_{j=1}^{k-1} \lambda_{j} \sum_{m=1}^{k} \frac{1}{\prod_{l=1, l \neq m}^{k}\left(\lambda_{l}-\lambda_{m}\right)} \frac{\sin \nu \pi}{\pi} \int_{0}^{\infty} d r \int_{0}^{\infty} \frac{r^{\nu-1} e^{-\lambda_{m}^{1 / \nu} z r}}{r^{2 \nu}+2 r^{\nu} \cos \nu \pi+1} q_{\nu}(t, z) d z \\
= & \prod_{j=1}^{k-1} \lambda_{j} \sum_{m=1}^{k} \frac{\sin \nu \pi}{\pi} \int_{0}^{\infty} \frac{r^{\nu-1} e^{-t\left(r \lambda_{m}^{1 / \nu}\right)^{\nu}}}{\prod^{2 \nu}+2 r^{\nu} \cos \nu \pi+1} d r \\
= & \int_{0}^{\infty} \operatorname{Pr}\left\{\mathcal{N}\left(t r^{\nu}\right)=k\right\} \frac{\sin \nu \pi}{\pi} \frac{r^{\nu-1}}{r^{2 \nu}+2 r^{\nu} \cos \nu \pi+1} d r \\
= & \operatorname{Pr}\left\{\mathcal{N}\left(t \mathcal{W}_{1}^{\nu}\right)=k\right\}=\operatorname{Pr}\left\{\mathcal{N}\left(t \mathcal{W}_{\nu}\right)=k\right\} .
\end{aligned}
$$


This concludes the proof of the first result. In order to prove the second result we write

$$
\begin{aligned}
& \operatorname{Pr}\left\{\mathcal{N}\left(\mathcal{S}^{\nu}\left(T_{2 \nu}(t)\right)\right)=k\right\} \\
& =\prod_{j=1}^{k-1} \lambda_{j} \sum_{m=1}^{k} \frac{1}{\prod_{l=1, l \neq m}^{k}\left(\lambda_{l}-\lambda_{m}\right)} \int_{0}^{\infty} \int_{0}^{\infty} e^{-\lambda_{m} s} q_{\nu}(z, s) f_{T_{2 \nu}}(z, t) d z d s \\
& =\prod_{j=1}^{k-1} \lambda_{j} \sum_{m=1}^{k} \frac{1}{\prod_{l=1, l \neq m}^{k}\left(\lambda_{l}-\lambda_{m}\right)} \int_{0}^{\infty} e^{-\lambda_{m}^{\nu} z} f_{T_{2 \nu}}(z, t) d z \\
& \text { by } \stackrel{(3.16)}{=} \prod_{j=1}^{k-1} \lambda_{j} \sum_{m=1}^{k} \frac{1}{\prod_{l=1, l \neq m}^{k}\left(\lambda_{l}-\lambda_{m}\right)} E_{\nu, 1}\left(-\lambda_{m}^{\nu} t^{\nu}\right) \\
& =\int_{0}^{\infty} \prod_{j=1}^{k-1} \lambda_{j} \sum_{m=1}^{k} \frac{1}{\prod_{l=1, l \neq m}^{k}\left(\lambda_{l}-\lambda_{m}\right)} \frac{\sin \nu \pi}{\pi} \frac{r^{\nu-1} e^{-\lambda_{m} t r}}{r^{2 \nu}+2 r^{\nu} \cos \nu \pi+1} d r \\
& =\int_{0}^{\infty} \operatorname{Pr}\{\mathcal{N}(t r)=k\} \frac{\sin \nu \pi}{\pi} \frac{r^{\nu-1}}{r^{2 \nu}+2 r^{\nu} \cos \nu \pi+1} d r=\operatorname{Pr}\left\{\mathcal{N}\left(t \mathcal{W}_{1}\right)=k\right\} .
\end{aligned}
$$

Remark 3.6. By slightly changing the above calculations, we arrive at the following result (compare with (3.49)):

$$
\operatorname{Pr}\left\{\mathcal{N}\left(\mathcal{S}^{\alpha}\left(T_{2 \nu}(t)\right)\right)=k\right\}=\prod_{j=1}^{k-1} \lambda_{j} \sum_{m=1}^{k} \frac{1}{\prod_{l=1, l \neq m}^{k}\left(\lambda_{l}-\lambda_{m}\right)} \mathbb{E} e^{-t \lambda_{m}^{\frac{\alpha}{\nu}} \mathcal{W}_{1}} .
$$

Remark 3.7. An alternative form of the distribution (3.49), for $\alpha=\nu$, can be given as follows.

$$
\begin{aligned}
& \operatorname{Pr}\left\{\mathcal{N}^{\nu}\left(\mathcal{S}^{\nu}(t)\right)=k\right\} \\
& =\prod_{j=1}^{k-1} \lambda_{j} \sum_{m=1}^{k} \frac{1}{\prod_{l=1, l \neq m}^{k}\left(\lambda_{l}-\lambda_{m}\right)} \frac{1}{2 i \nu \pi} \int_{0}^{\infty}\left(\frac{1}{w+e^{-i \pi \nu}}-\frac{1}{w+e^{i \pi \nu}}\right) e^{-t \lambda_{m} w} d w \\
& =\prod_{j=1}^{k-1} \lambda_{j} \sum_{m=1}^{k} \frac{1}{\prod_{l=1, l \neq m}^{k}\left(\lambda_{l}-\lambda_{m}\right)} \frac{1}{2 i \nu \pi}\left[e^{t \lambda_{m} e^{-i \pi \nu}} \mathrm{E}_{1}\left(t \lambda_{m} e^{-i \pi \nu}\right)-e^{t \lambda_{m} e^{i \pi \nu}} \mathrm{E}_{1}\left(t \lambda_{m} e^{i \pi \nu}\right)\right] \\
& =\prod_{j=1}^{k-1} \lambda_{j} \sum_{m=1}^{k} \frac{1}{\prod_{l=1, l \neq m}^{k}\left(\lambda_{l}-\lambda_{m}\right)} \frac{e^{\lambda_{m} t \cos \nu \pi}}{2 i \nu \pi}\left[e^{t \lambda_{m} \sin \nu \pi} \mathrm{E}_{1}\left(t \lambda_{m} e^{-i \pi \nu}\right)-e^{t \lambda_{m} \sin \nu \pi} \mathrm{E}_{1}\left(t \lambda_{m} e^{i \pi \nu}\right)\right],
\end{aligned}
$$

where the function $\mathrm{E}_{1}(z)=\int_{z}^{\infty} \frac{e^{-t}}{t} d t,|\arg z<\pi|$, is the exponential integral.

\subsection{Fractional pure birth process stopped at $T_{2 \alpha}(t)$}

In this section we consider the process $\mathcal{N}^{\nu}\left(T_{2 \alpha}(t)\right), t>0$ (see the discussion related to formula (3.6). As we did before, here we treat the case $k \geq 2$. The state probabilities can be written as follows.

$$
\begin{aligned}
p_{k}^{\nu, \alpha}(t) & =\operatorname{Pr}\left\{\mathcal{N}^{\nu}\left(T_{2 \alpha}(t)\right)=k \mid \mathcal{N}^{\nu}(0)=1\right\} \\
& =\prod_{j=1}^{k-1} \lambda_{j} \sum_{m=1}^{k} \frac{1}{\prod_{l=1, l \neq m}^{k}\left(\lambda_{l}-\lambda_{m}\right)} \int_{0}^{\infty} E_{\nu, 1}\left(-\lambda_{m} s^{\nu}\right) \operatorname{Pr}\left\{T_{2 \alpha}(t) \in d s\right\}
\end{aligned}
$$


The integral in (3.66) can be further worked out by means of the Laplace transform:

$$
\begin{aligned}
& \int_{0}^{\infty} E_{\nu, 1}\left(-\lambda_{m} s^{\nu}\right) \int_{0}^{\infty} e^{-z t} \operatorname{Pr}\left\{T_{2 \alpha}(t) \in d s\right\} d t \\
& =\int_{0}^{\infty} E_{\nu, 1}\left(-\lambda_{m} s^{\nu}\right) z^{\alpha-1} e^{-s z^{\alpha}} d s=z^{\alpha-1} \frac{\left(z^{\alpha}\right)^{\nu-1}}{z^{\alpha \nu}+\lambda_{m}}=\frac{z^{\alpha \nu-1}}{z^{\alpha \nu}+\lambda_{m}} .
\end{aligned}
$$

By taking the inverse Laplace transform of the above formula, we immediately obtain that

$$
\int_{0}^{\infty} E_{\nu, 1}\left(-\lambda_{m} s^{\nu}\right) \operatorname{Pr}\left\{T_{2 \alpha}(t) \in d s\right\}=\int_{0}^{\infty} E_{\nu, 1}\left(-\lambda_{m} s^{\nu}\right) t^{-\alpha} W_{-\alpha, 1-\alpha}\left(-t^{-\alpha} s\right) d s=E_{\nu \alpha, 1}\left(-\lambda_{m} t^{\nu \alpha}\right) .
$$

Therefore, the state probabilities for the process $\mathcal{N}^{\nu}\left(T_{2 \alpha}(t)\right), t>0$, result in the following form:

$$
p_{k}^{\nu, \alpha}(t)=\prod_{j=1}^{k-1} \lambda_{j} \sum_{m=1}^{k} \frac{1}{\prod_{l=1, l \neq m}^{k}\left(\lambda_{l}-\lambda_{m}\right)} E_{\nu \alpha, 1}\left(-\lambda_{m} t^{\nu \alpha}\right)=p_{k}^{\nu \alpha}(t), \quad k \geq 2 .
$$

Note that the case $k=1$ can be treated in the same manner. We thus obtain the following equalities in distribution:

$$
\mathcal{N}^{\nu}\left(T_{2 \alpha}(t)\right)=\mathcal{N}\left\{T_{2 \nu}\left(T_{2 \alpha}(t)\right)\right\}=\mathcal{N}\left(T_{2 \nu \alpha}(t)\right)=\mathcal{N}^{\nu \alpha}(t), \quad t>0 .
$$

Let now $\eta_{n}=\prod_{i=1}^{n} \nu_{i}$, where $n \in \mathbb{N}$, and $\nu_{i}$ are $n$ indices such that $\nu_{i} \in(0,1]$ for $1 \leq i \leq n$. Formula (3.70) can be generalised as

$$
\mathcal{N}\left\{T_{2 \nu_{1}}\left(T_{2 \nu_{2}}\left(\ldots T_{2 \nu_{n}}(t) \ldots\right)\right)\right\}=\mathcal{N}\left(T_{2 \eta_{n}}(t)\right)=\mathcal{N}^{\eta_{n}}(t), \quad t>0
$$

where $\mathfrak{N}^{\eta_{n}}(t)$ is a nonlinear fractional birth process.

Formula (3.66) can also be worked out in an alternative way. In the following calculations we will make use of the integral representation (3.5).

$$
\begin{aligned}
p_{k}^{\nu, \alpha}(t)= & \operatorname{Pr}\left\{\mathcal{L}^{\nu}\left(T_{2 \alpha}(t)\right)=k \mid \mathcal{N}^{\nu}(0)=1\right\} \\
= & \prod_{j=1}^{k-1} \lambda_{j} \sum_{m=1}^{k} \frac{1}{\prod_{l=1, l \neq m}^{k}\left(\lambda_{l}-\lambda_{m}\right)} \int_{0}^{\infty} E_{\nu, 1}\left(-\lambda_{m} s^{\nu}\right) \operatorname{Pr}\left\{T_{2 \alpha}(t) \in d s\right\} \\
= & \int_{0}^{\infty} \prod_{j=1}^{k-1} \lambda_{j} \sum_{m=1}^{k} \frac{1}{\prod_{l=1, l \neq m}^{k}\left(\lambda_{l}-\lambda_{m}\right)} \\
& \times \int_{0}^{\infty} \frac{\sin \nu \pi}{\pi} \frac{r^{\nu-1}}{r^{2 \nu}+2 r^{\nu} \cos \nu \pi+1} e^{-r \lambda_{m}^{\frac{1}{\nu}} s} \operatorname{Pr}\left\{T_{2 \alpha}(t) \in d s\right\} d r \\
= & \prod_{j=1}^{k-1} \lambda_{j} \sum_{m=1}^{k} \frac{1}{\prod_{l=1, l \neq m}^{k}\left(\lambda_{l}-\lambda_{m}\right)} \int_{0}^{\infty} \frac{\sin \nu \pi}{\pi} \frac{r^{\nu-1}}{r^{2 \nu}+2 r^{\nu} \cos \nu \pi+1} E_{\alpha, 1}\left(-r \lambda_{m}^{\frac{1}{\nu}} t^{\alpha}\right) .
\end{aligned}
$$

Remark 3.8. By comparing formulae (3.69) and (3.72), it is clear that the following expansion holds:

$$
E_{\nu \alpha, 1}\left(-\lambda_{m} t^{\nu \alpha}\right)=\frac{\sin \nu \pi}{\pi} \int_{0}^{\infty} \frac{r^{\nu-1}}{r^{2 \nu}+2 r^{\nu} \cos \nu \pi+1} E_{\alpha, 1}\left(-r \lambda_{m}^{\frac{1}{\nu}} t^{\alpha}\right) d r, \quad \nu \in(0,1], \alpha \in(0,1] .
$$

We give a direct proof of (3.73) by applying the Laplace transform to both members. Of course

$$
\int_{0}^{\infty} e^{-\mu t} E_{\nu \alpha, 1}\left(-\lambda_{m} t^{\nu \alpha}\right) d t=\frac{\mu^{\nu \alpha-1}}{\mu^{\nu \alpha}+\lambda_{m}} .
$$


Then, we must calculate the twofold integral

$$
\begin{aligned}
& \frac{\sin \nu \pi}{\pi} \int_{0}^{\infty} e^{-\mu t} \int_{0}^{\infty} \frac{r^{\nu-1}}{r^{2 \nu}+2 r^{\nu} \cos \nu \pi+1} E_{\alpha, 1}\left(-r \lambda_{m}^{\frac{1}{\nu}} t^{\alpha}\right) d t d r \\
& =\frac{\sin \nu \pi}{\pi} \int_{0}^{\infty} \frac{r^{\nu-1}}{r^{2 \nu}+2 r^{\nu} \cos \nu \pi+1} \frac{\mu^{\alpha-1}}{\mu^{\alpha}+r \lambda_{m}^{\frac{1}{\nu}}} d r \\
& =\frac{\sin \nu \pi}{\pi} \int_{0}^{\infty} \int_{0}^{\infty} \frac{\mu^{\alpha-1} r^{\nu-1} e^{-w\left(\mu^{\alpha}+r \lambda_{m}^{\frac{1}{\nu}}\right)}}{r^{2 \nu}+2 r^{\nu} \cos \nu \pi+1} d r d w \\
& b y \stackrel{(3.5)}{=} \int_{0}^{\infty} \mu^{\alpha-1} e^{-\mu^{\alpha} w} E_{\nu, 1}\left(-\lambda_{m} w^{\nu}\right) d w=\frac{\left(\mu^{\alpha}\right)^{\nu-1} \mu^{\alpha-1}}{\left(\mu^{\alpha}\right)^{\nu}+\lambda_{m}}=\frac{\mu^{\nu \alpha-1}}{\mu^{\nu \alpha}+\lambda_{m}} .
\end{aligned}
$$

Remark 3.9. A number of interesting relations follow from formula (3.73).

The following integral relation holds:

$$
\begin{aligned}
& \frac{\sin \nu \pi}{\nu} \int_{0}^{\infty} \frac{1}{r^{2}+2 r \cos \nu \pi+1} E_{\alpha, 1}\left(-r^{\frac{1}{\nu}} \lambda_{m}^{\frac{1}{\nu}} t^{\alpha}\right) d r \\
& =\frac{\sin \nu \alpha \pi}{\nu \alpha} \int_{0}^{\infty} \frac{1}{r^{2}+2 r \cos \nu \alpha \pi+1} e^{-r \frac{1}{\nu \alpha} \lambda_{m}^{\frac{1}{\nu \alpha}} t} d r .
\end{aligned}
$$

A sort of commutativity is valid for (3.73):

$$
E_{\alpha \nu, 1}\left(-\lambda_{m} t^{\alpha \nu}\right)=\frac{\sin \alpha \pi}{\pi} \int_{0}^{\infty} \frac{r^{\alpha-1}}{r^{2 \alpha}+2 r^{\alpha} \cos \alpha \pi+1} E_{\nu, 1}\left(-r \lambda_{m}^{\frac{1}{\alpha}} t^{\nu}\right), \quad \nu \in(0,1], \alpha \in(0,1] .
$$

For $\alpha=1$ we recover, from (3.73), the integral representation of Mittag-Leffler functions. By considering that

$$
f_{\nu}(r)=\frac{r^{\nu-1}}{r^{2 \nu}+2 r^{\nu} \cos \nu \pi+1},
$$

for $\nu=1$, becomes a delta function with pole at $r=1$, we extract, from (3.73), an identity.

Furthermore, it is worth noticing that formulae similar to (3.73) can be derived by repeated applications of the same formula. For example we have:

$$
\begin{aligned}
& E_{\nu \alpha \beta, 1}\left(-\lambda_{m} t^{\nu \alpha \beta}\right)=\frac{\sin \nu \pi}{\pi} \int_{0}^{\infty} \frac{r^{\nu-1}}{r^{2 \nu}+2 r^{\nu} \cos \nu \pi+1} E_{\alpha \beta, 1}\left(-r \lambda_{m}^{\frac{1}{\nu}} t^{\alpha \beta}\right) d r \\
& =\frac{\sin \nu \pi \sin \alpha \pi}{\pi^{2}} \int_{0}^{\infty} \int_{0}^{\infty} \frac{r^{\nu-1} w^{\alpha-1} E_{\beta, 1}\left(-w r^{\frac{1}{\alpha}} \lambda_{m}^{\frac{1}{\nu \alpha}} t^{\beta}\right)}{\left(r^{2 \nu}+2 r^{\nu} \cos \nu \pi+1\right)\left(w^{2 \alpha}+2 w^{\alpha} \cos \alpha \pi+1\right)} d w d r .
\end{aligned}
$$

Let $\nu_{i}, 1 \leq i \leq n$ be $n$ indices such that for all $1 \leq i \leq n, \nu_{i} \in(0,1]$, and let us denote $\eta_{n}=\prod_{i=1}^{n} \nu_{i}$. In general, for $n \geq 2$, we obtain that

$$
\begin{aligned}
& E_{\eta_{n}, 1}\left(-\lambda_{m} t^{\eta_{n}}\right) \\
& =\int_{0}^{\infty} \ldots \int_{0}^{\infty} \prod_{j=1}^{n-1}\left(\frac{r_{j}^{\nu_{j}-1}}{r_{j}^{2 \nu_{j}}+2 r_{j}^{\nu_{j}} \cos \nu_{j}+1}\right) E_{\nu_{n}, 1}\left(-r_{1} r_{2}^{\frac{1}{\nu_{1}}} r_{3}^{\frac{1}{\nu_{1} \nu_{2}}} \ldots r_{n-1}^{\frac{1}{\eta_{n}-2}} \lambda_{m}^{\frac{1}{\eta_{n}-1}} t^{\nu_{n}}\right) \prod_{j=1}^{n-1} d r_{j} \\
& =\mathbb{E}\left[E_{\nu_{n}, 1}\left(-{ }^{(1)} \mathcal{W}_{1}{ }^{(2)} \mathcal{W}_{1}^{\frac{1}{\nu_{1}}(3)} \mathcal{W}_{1}^{\frac{1}{\nu_{1} \nu_{2}}} \ldots{ }^{\left({ }^{n-1)}\right.} \mathcal{W}_{1}^{\frac{1}{\eta_{n}-2}} \lambda_{m}^{\frac{1}{\eta_{n-1}}} t^{\nu_{n}}\right)\right],
\end{aligned}
$$

where ${ }^{(j)} \mathcal{W}_{1}, 1 \leq j \leq n-1$, are independent random variables, each with distribution (3.50), with $\alpha=1$ and $\nu=\nu_{j}$. 


\section{References}

[1] M. Abramowitz and I. A. Stegun. Handbook of Mathematical Functions with Formulas, Graphs, and Mathematical Tables. Dover Publications, Inc., New York, ninth dover printing, tenth gpo printing edition, 1964.

[2] B. Baeumer, M. M. Meerschaert, and E. Nane. Space-time duality for fractional diffusion. J. Appl. Probab., 46(4):1100-1115, 2009.

[3] L. Beghin and E. Orsingher. Fractional Poisson processes and related planar random motions. Electron. J. Probab., 14(61):1790-1826, 2009.

[4] S. Bochner. Harmonic Analysis and the Theory of Probability. University of California Press, Berkeley, 1955.

[5] C. L. Chiang. Introduction to Stochastic Processes in Biostatistics.

[6] W. Feller. An Introduction to Probability Theory and Its Applications, Volume 1. Wiley \& Sons, New York, third edition, 1968.

[7] I. I. Gikhman and A. V. Skorokhod. Introduction to the Theory of Random Processes. Dover Publications, New York, 1996.

[8] G. R. Grimmett and D. R. Stirzaker. Probability and Random Processes. Oxford University Press, 2001.

[9] A. A. Kilbas, H. M. Srivastava, and J. J. Trujillo. Theory and Applications of Fractional Differential Equations. Elsevier Science, 2006.

[10] J. Lamperti. An occupation time theorem for a class of stochastic processes. Tran. Amer. Math. Soc., 88(2):380-387, 1958 .

[11] N. Laskin. Fractional Poisson process. Commun. Nonlinear Sci. Numer. Simul., 8(3-4):201-213, 2003.

[12] M.-L. T. Lee and G. A. Whitmore. Stochastic Processes Directed by Randomized Time. J. Appl. Probab., 30(2):302-314, 1993.

[13] F. Mainardi and R. Gorenflo. A fractional generalization of the Poisson processes. Vietnam J. Math., 32:53-64, 2004.

[14] M. M. Meerschaert, E. Nane, and P. Vellaisamy. The fractional Poisson process and the inverse stable subordinator. arXiv:1007.5051v1 [math.PR], 2010.

[15] E. Orsingher and F. Polito. Fractional pure birth processes. Bernoulli, 16(3):858-881, 2010.

[16] E. Orsingher, F. Polito, and L. Sakhno. Fractional Non-Linear, Linear and Sublinear Death Processes. J. Stat. Phys., 141(1):68-93, 2010.

[17] V. V. Uchaikin, D. O. Cahoy, and R. T. Sibatov. Fractional Processes: From Poisson to Branching One. Internat. J. Bifur. Chaos Appl. Sci. Engrg., 18(9):2717-2725, 2008. 